TRANSACTIONS OF THE

AMERICAN MATHEMATICAL SOCIETY

Volume 350, Number 2, February 1998, Pages 769-808

S 0002-9947(98)01879-0

\title{
CONTIGUOUS RELATIONS, CONTINUED FRACTIONS AND ORTHOGONALITY
}

\author{
DHARMA P. GUPTA AND DAVID R. MASSON
}

\begin{abstract}
We examine a special linear combination of balanced very-wellpoised ${ }_{10} \phi 9$ basic hypergeometric series that is known to satisfy a transformation. We call this $\Phi$ and show that it satisfies certain three-term contiguous relations. From two of these contiguous relations for $\Phi$ we obtain fifty-six pairwise linearly independent solutions to a three-term recurrence that generalizes the recurrence for Askey-Wilson polynomials. The associated continued fraction is evaluated using Pincherle's theorem. From this continued fraction we are able to derive a discrete system of biorthogonal rational functions. This ties together Wilson's results for rational biorthogonality, Watson's $q$-analogue of Ramanujan's Entry 40 continued fraction, and a conjecture of Askey concerning the latter. Some new $q$-series identities are also obtained. One is an important three-term transformation for $\Phi$ 's which generalizes all the known two- and three-term ${ }_{8} \phi_{7}$ transformations. Others are new and unexpected quadratic identities for these very-well-poised $8 \phi_{7}$ 's.
\end{abstract}

\section{INTRODUCTION}

Background. It is now just over ten years since Askey-Wilson polynomials were introduced as an explicit system of orthogonal polynomials that generalize Jacobi polynomials [2]. Here we are concerned with a generalization of Askey-Wilson polynomials which yields an explicit system of biorthogonal rational functions. This generalization was first given by Wilson in [31] and later by Rahman and Suslov $[26]$.

The explicitness of the above orthogonalities is connected to the fact that certain hypergeometric and basic hypergeometric series satisfy three-term contiguous relations. The importance of contiguous relations in this context was emphasized by Wilson in his thesis [30]. For more recent work which makes extensive use of contiguous relations, see [16], [23], [13], [14], [15].

In the case of Askey-Wilson polynomials, the relevant series are a terminating, balanced ${ }_{4} \phi_{3}$ series, which describes the polynomials themselves [2], and a very-wellpoised ${ }_{8} \phi_{7}$ series, which describes the minimal solution to the polynomial recurrence [18], [11].

The necessity of a three-term contiguous relation is dictated by the fact that an orthogonal polynomial system $\left\{P_{n}(x)\right\}_{n=0}^{\infty}$ must satisfy a three-term recurrence of

Received by the editors November 21, 1995 and, in revised form, July 2, 1996.

1991 Mathematics Subject Classification. Primary 33D45, 40A15, 39A10, 47B39.

Key words and phrases. Contiguous relations, difference equations, minimal solution, continued fractions, biorthogonal rational functions, three-term-transformation, quadratic identities.

Research partially supported by NSERC (Canada). 
the form $[6]$

$$
P_{n+1}(x)-\left(x-c_{n+1}\right) P_{n}(x)+\lambda_{n+1} P_{n-1}(x)=0,
$$

associated with the $J$-fraction

$$
\frac{1}{x-c_{1}}-\frac{\lambda_{2}}{x-c_{2}}-\frac{\lambda_{3}}{x-c_{3}}-\ldots .
$$

Are there explicit orthogonal polynomial systems more general than AskeyWilson polynomials? The answer is believed to be no, since there are no known basic hypergeometric series which are more general and which also satisfy a threeterm contiguous relation that can be cast into the form (1.1).

However, if we relax the requirement that the orthogonal system consists of polynomials, then we are led to Wilson's system of biorthogonal rational functions [31]. With only a slight modification this again seems to be the most general model of its type.

In this paper we examine this general rational function biorthogonality by starting with some three-term contiguous relations of the most general known type. The basic hypergeometric series involved are terminating, balanced, very-well-poised ${ }_{10} \phi_{9}$ 's or, more generally, special linear combinations of non-terminating such ${ }_{10} \phi_{9}$ 's which we call $\Phi$ 's. Both of these satisfy three-term contiguous relations which can be put into the form

$$
P_{n+1}(x)-\left(x-c_{n+1}\right) P_{n}(x)+\lambda_{n+1}\left(x-\alpha_{n+1}\right)\left(x-\beta_{n+1}\right) P_{n-1}(x)=0
$$

associated with the $R_{I I}$-fraction

$$
\frac{1}{x-c_{1}}-\frac{\lambda_{2}\left(x-\alpha_{2}\right)\left(x-\beta_{2}\right)}{x-c_{2}}-\frac{\lambda_{3}\left(x-\alpha_{3}\right)\left(x-\beta_{3}\right)}{x-c_{3}}-\ldots .
$$

That the orthogonal systems corresponding to (1.3) and (1.4) involve rational functions rather than polynomials has been demonstrated by Ismail and Masson [17].

There is a second related aspect which lead us to examine this most general basic hypergeometric level. Many of the continued fractions of Ramanujan are connected with orthogonal polynomials. Some of the most intriguing of these are expressed in terms of gamma functions [5] and have been shown to be connected with special limiting cases of Askey-Wilson polynomials [21], [22], [9]. Armed with this fact, we were able to extend and give new meaning to Ramanujan's famous Entry 40 [24] and its $q$-analogue given by Watson [29], [10]. These are all special cases of the very general continued fraction we examine in Section 3 of this paper. From the simplest terminating form of this continued fraction given in Corollary 3.3 and the orthogonality derived in Section 4, we are now able to vindicate Askey's conjecture $\left[1\right.$, p. 37] that Ramanujan's Entry 40 is connected with Dougall's ${ }_{7} F_{6}$ summation formula and a three-term recurrence for a very-well-poised ${ }_{9} F_{8}$.

Results. In Section 2 we prove that $\Phi$ satisfies certain three-term contiguous relations. For two of these (Theorems 2.4 and 2.5) we make essential use of the two-term transformation formula for $\Phi$ [7, (III. 39), p. 247]. This generalizes our earlier work in [10].

In Section 3, Theorems 2.4 and 2.5 are used to obtain fifty-six pairwise linearly independent solutions to a very general eight parameter three-term recurrence. This recurrence generalizes the recurrence for Askey-Wilson polynomials. The large $n$ asymptotics of the solutions is also examined. From two of these solutions we are then able to construct a minimal solution. This minimal solution, via Pincherle's 
Theorem, gives an explicit but complicated continued fraction result which generalizes Ramanujan's Entry 40 and our earlier work [10]. Two special cases are considered. One is a terminating fraction given in terms of a terminating ${ }_{10} \phi_{9}[28]$.

In Section 4 this terminating fraction is expressed as an $R_{I I}$-fraction and used to derive a very general explicit rational biorthogonality. This derivation follows the methods in [17]. Also in Section 4 we give six limiting cases of this biorthogonality. Five limits are at the ${ }_{4} \phi_{3}$ or ${ }_{8} \phi_{7}$ level and one is a $q \rightarrow 1$ limit at the ${ }_{9} F_{8}$ level. One of the ${ }_{4} \phi_{3}$ limits corresponds to the case of $q$-Racah polynomials, while the other orthogonalities are new. By taking further limits this can be extended to a full Askey-type scheme of rational biorthogonality. For a detailed review of the Askey-scheme for polynomial orthogonality, see Koekoek and Swarttouw [20].

In Section 5 we give further details for one of the limit cases at the very-wellpoised ${ }_{8} \phi_{7}$ level. This expands on some of our previous results for this model [12]. Also a general three-term ${ }_{8} \phi_{7}$ transformation is derived which is essential for Section 6 .

We have already mentioned that $\Phi$ satisfies a two-term transformation formula. This is the most general two-term transformation given in Gasper and Rahman [7]. In Section 6 we derive a missing companion transformation. This is a three-term $\Phi$ transformation which generalizes all the known ${ }_{8} \phi_{7}$ transformations.

Finally, in Section 7 we derive what we feel are some unexpected ${ }_{8} \phi_{7}$ identities. These new quadratic identities are derived from the asymptotics of the Casorati determinants of some of the recurrence solutions of Section 3.

Notation. We follow the notation in Gasper and Rahman [7] except that we omit the designation ' $q$ ' for the base in the $q$-shifted factorials and the basic hypergeometric functions. Thus, given a number $q$, the $q$-shifted factorial is defined by

$$
\begin{aligned}
& (a)_{0}:=1, \quad(a)_{n}:=\prod_{j=1}^{n}\left(1-a q^{j-1}\right), \quad n=1,2, \ldots, \\
& (a)_{-n}:=\frac{1}{\left(a q^{-n}\right)_{n}}, \quad n=1,2, \ldots,
\end{aligned}
$$

and for $|q|<1$,

$$
(a)_{\infty}=\prod_{j=1}^{\infty}\left(1-a q^{j-1}\right) .
$$

We also write

$$
\left(a_{1}, a_{2}, \ldots, a_{k}\right)_{n}:=\prod_{j=1}^{k}\left(a_{j}\right)_{n}, \quad n \text { integer or } n=\infty .
$$

The ${ }_{r} \phi_{s}$ basic hypergeometric series is given by

$$
{ }_{r} \phi_{s}\left(\begin{array}{c}
a_{1}, a_{2}, \ldots, a_{r} \\
b_{1}, b_{2}, \ldots, b_{s}
\end{array} z\right):=\sum_{n=0}^{\infty} \frac{\left(a_{1}, a_{2}, \ldots, a_{r}\right)_{n}}{\left(b_{1}, b_{2}, \ldots, b_{s}, q\right)_{n}}\left[(-1)^{n} q^{\left(\begin{array}{c}
n \\
2
\end{array}\right)}\right]^{1+s-r} z^{n},
$$

and the general bilateral basic hypergeometric series is defined by

$$
{ }_{r} \psi_{s}\left(\begin{array}{c}
a_{1}, a_{2}, \ldots, a_{r} \\
b_{1}, b_{2}, \ldots, b_{s}
\end{array} ;\right):=\sum_{n=-\infty}^{\infty} \frac{\left(a_{1}, a_{2}, \ldots, a_{r}\right)_{n}}{\left(b_{1}, b_{2}, \ldots, b_{s}\right)_{n}}\left[(-1)^{n} q^{\left.\left(\begin{array}{c}
n \\
2
\end{array}\right)\right]^{s-r}} z^{n} .\right.
$$


We denote a very-well-poised, balanced ${ }_{10} \phi_{9}$ series as

$$
\begin{aligned}
& \phi={ }_{10} W_{9}(a ; b, c, d, e, f, g, h ; q):={ }_{10} \phi_{9}\left(\begin{array}{c}
a, q \sqrt{a},-q \sqrt{a}, b, c, d, e, f, g, h \\
\left.\sqrt{a},-\sqrt{a}, \frac{a q}{b}, \frac{a q}{c}, \frac{a q}{d}, \frac{a q}{e}, \frac{a q}{f}, \frac{a q}{g}, \frac{a q}{h} ; q\right),
\end{array}\right. \\
& \text { bcdefgh }=a^{3} q^{2},
\end{aligned}
$$

and the limiting case of a very-well-poised ${ }_{8} \phi_{7}$ as

$$
{ }_{8} W_{7}\left(a ; b, c, d, e, f ; \frac{a^{2} q^{2}}{b c d e f}\right):={ }_{8} \phi_{7}\left(\begin{array}{c}
a, q \sqrt{a},-q \sqrt{a}, b, c, d, e, f \\
\sqrt{a},-\sqrt{a}, \frac{a q}{b}, \frac{a q}{c}, \frac{a q}{d}, \frac{a q}{e}, \frac{a q}{f} ; \frac{a^{2}}{b c d e f}
\end{array}\right) .
$$

A complementary pair of very-well-poised, balanced ${ }_{10} \phi_{9}$ 's is defined by

$$
\begin{aligned}
& \Phi^{(b)}(a ; b, c, d, e, f, g, h ; q) \\
&:={ }_{10} W_{9}(a ; b, c, d, e, f, g, h ; q) \\
&+ \frac{\left(a q, \frac{b}{a}, c, d, e, f, g, h, \frac{b q}{c}, \frac{b q}{d}, \frac{b q}{e}, \frac{b q}{f}, \frac{b q}{g}, \frac{b q}{h}\right)_{\infty}}{\left(\frac{b^{2} q}{a}, \frac{a}{b}, \frac{a q}{c}, \frac{a q}{d}, \frac{a q}{e}, \frac{a q}{f}, \frac{a q}{g}, \frac{a q}{h}, \frac{b c}{a}, \frac{b d}{a}, \frac{b e}{a}, \frac{b f}{a}, \frac{b g}{a}, \frac{b h}{a}\right)_{\infty}} \\
& \times{ }_{10} W_{9}\left(\frac{b^{2}}{a} ; b, \frac{b c}{a}, \frac{b d}{a}, \frac{b e}{a}, \frac{b f}{a}, \frac{b g}{a}, \frac{b h}{a} ; q\right)=\phi+\phi^{\prime(b)},
\end{aligned}
$$

say, with bcdefgh $=a^{3} q^{2}$. Here, ' $b$ ' is a distinguished parameter which can be interchanged with $c, d, e, f, g$ or $h$ to give different $\Phi$ 's. We use the notation

$$
\Phi=\Phi(a ; b, c, d, e, f, g, h ; q)=\phi+\phi^{\prime}
$$

to denote any one of these seven possible complementary pairs. Whenever we want to specify the distinguished parameter ' $b$ ' while defining $\Phi$ or $\phi^{\prime}$, we shall denote them by $\Phi^{(b)}$ and $\phi^{(b)}$ respectively.

We will avoid singularities in the definition of $\Phi^{(b)}$. In particular, we assume that $a \neq b$. If any one of the parameters $c, d, e, f, g, h$ is $q^{-n}, n=0,1, \cdots$, the complementary part $\phi^{(b)}$ vanishes.

A $q$-analogue of Wilson's notation [29] will be used for the variations of $\phi, \Phi$ or $\phi^{\prime}$ with respect to the parameters. Thus, $\Phi^{(b)}(g+, h-)$ represents the expression (1.8) with ' $g$ ' and ' $h$ ' replaced throughout by ' $g q$ ' and ' $\frac{h}{q}$ ' respectively. $\Phi^{(b)} \pm$ denotes the expression which would be obtained by replacements

$$
(a, b, c, d, e, f, g, h) \rightarrow\left(a q^{ \pm 2}, b q^{ \pm 1}, c q^{ \pm 1}, d q^{ \pm 1}, e q^{ \pm 1}, f q^{ \pm 1}, g q^{ \pm 1}, h q^{ \pm 1}\right)
$$

throughout the expression (1.8).

\section{Contiguous Relations}

We obtain three-term contiguous relations for the $\Phi$ function which generalize the relations we obtained earlier [10] for ${ }_{10} \phi_{9}$ 's. The method of proof is basically the same as the one employed by Wilson [29] in his study of ${ }_{9} F_{8}$ functions and employed by us in our previous work [10]. 
Lemma 2.1. [10, p. 431, Lemma 1] If $\phi$ denotes the balanced very-well-poised ${ }_{10} \phi_{9}$ series defined by (1.7), then

$$
\begin{aligned}
& \phi(g-, h+)-\phi \\
& =\frac{\frac{a q}{h}\left(1-\frac{h q}{g}\right)\left(1-\frac{g h}{a q}\right)(1-a q)\left(1-a q^{2}\right)(1-b)(1-c)}{\left(1-\frac{a q}{g}\right)\left(1-\frac{a q^{2}}{g}\right)\left(1-\frac{a}{h}\right)\left(1-\frac{a q}{h}\right)\left(1-\frac{a q}{b}\right)\left(1-\frac{a q}{c}\right)} \\
& \quad \times \frac{(1-d)(1-e)(1-f)}{\left(1-\frac{a q}{d}\right)\left(1-\frac{a q}{e}\right)\left(1-\frac{a q}{f}\right)} \phi_{+}(g-) .
\end{aligned}
$$

Lemma 2.2. If $\phi$ denotes the balanced very-well-poised ${ }_{10} \phi_{9}$ series defined by (1.7), then

$$
\begin{gathered}
c(1-c)\left(1-\frac{a}{c}\right)\left(1-\frac{d q}{g}\right)\left(1-\frac{g d}{a q}\right) \phi(g-, c+) \\
-d(1-d)\left(1-\frac{a}{d}\right)\left(1-\frac{c q}{g}\right)\left(1-\frac{g c}{a q}\right) \phi(g-, d+) \\
+d\left(1-\frac{g}{q}\right)\left(1-\frac{c}{d}\right)\left(1-\frac{a q}{g}\right)\left(1-\frac{c d}{a}\right) \phi=0 .
\end{gathered}
$$

Proof. Elimination of $\phi_{+}(g-)$ from two relations written for $\phi(g-, c+)-\phi$ and $\phi(g-, d+)-\phi$ with the help of Lemma 2.1 gives the required result.

Lemma 2.1'. Irrespective of the choice of the distinguished parameter, $\Phi$ satisfies the relation

$$
\begin{aligned}
& \Phi(g-, h+)-\Phi \\
& =\frac{\frac{a q}{h}\left(1-\frac{h q}{g}\right)\left(1-\frac{g h}{a q}\right)(1-a q)\left(1-a q^{2}\right)(1-b)(1-c)}{\left(1-\frac{a q}{g}\right)\left(1-\frac{a q^{2}}{g}\right)\left(1-\frac{a}{h}\right)\left(1-\frac{a q}{h}\right)\left(1-\frac{a q}{b}\right)\left(1-\frac{a q}{c}\right)} \\
& \quad \times \frac{(1-d)(1-e)(1-f)}{\left(1-\frac{a q}{d}\right)\left(1-\frac{a q}{e}\right)\left(1-\frac{a q}{f}\right)} \Phi_{+}(g-) .
\end{aligned}
$$

Proof. In view of Lemma 2.1, we only need to prove that (2.3) holds true for the complementary part $\phi^{\prime}$ irrespective of the choice of the distinguished parameter. It is evident that we should check the validity of the statement in three different cases, viz., when the distinguished parameter is either ' $g$ ' or ' $h$ ' or it is one of the parameters $b, c, d, e$ or $f$.

The statement (2.3) can be shown to be true for $\phi^{(g)}$ if we apply Lemma 2.1 to the series

$$
{ }_{10} W_{9}\left(\frac{g^{2}}{a q^{2}} ; \frac{g b}{a q}, \frac{g c}{a q}, \frac{g d}{a q}, \frac{g e}{a q}, \frac{g f}{a q}, g, \frac{g h}{a q} ; q\right)
$$

and make the required simplification.

Similarly, validity of (2.3) for $\phi^{(h)}$ can be derived by applying Lemma 2.1 to

$$
{ }_{10} W_{9}\left(\frac{h^{2}}{a} ; \frac{h b}{a}, \frac{h c}{a}, \frac{h d}{a}, \frac{h e}{a}, \frac{h f}{a}, \frac{h g}{a}, h ; q\right) .
$$

In the third case, say for example for ${\phi^{(}}^{(b)}$, we can apply (2.2) of Lemma 2.2 to the function

$$
{ }_{10} W_{9}\left(\frac{b^{2}}{a} ; b, \frac{b c}{a}, \frac{b d}{a}, \frac{b e}{a}, \frac{b f}{a}, \frac{b g}{a}, \frac{b h}{a} ; q\right)
$$


and we arrive at the desired result. This completes the proof of the lemma.

In view of Lemma 2.2 and Lemma $2.1^{\prime}$ we can immediately state Lemma $2.2^{\prime}$.

Lemma 2.2'. Irrespective of the choice of the distinguished parameter, $\Phi$ satisfies the relation

$$
\begin{gathered}
c(1-c)\left(1-\frac{a}{c}\right)\left(1-\frac{d q}{g}\right)\left(1-\frac{g d}{a q}\right) \Phi(g-, c+) \\
-d(1-d)\left(1-\frac{a}{d}\right)\left(1-\frac{c q}{g}\right)\left(1-\frac{g c}{a q}\right) \Phi(g-, d+) \\
+d\left(1-\frac{g}{q}\right)\left(1-\frac{c}{d}\right)\left(1-\frac{a q}{g}\right)\left(1-\frac{c d}{a}\right) \Phi=0
\end{gathered}
$$

Theorem 2.3. Irrespective of the choice of the distinguished parameter, $\Phi$ satisfies the relation

$$
\begin{gathered}
\frac{g^{2}(1-h)\left(1-\frac{a q}{g b}\right)\left(1-\frac{a q}{g c}\right)\left(1-\frac{a q}{g d}\right)\left(1-\frac{a q}{g e}\right)\left(1-\frac{a q}{g f}\right)}{\left(1-\frac{a q}{g}\right)\left(1-\frac{a q^{2}}{g}\right)} \Phi_{+}(g-) \\
-\frac{h^{2}(1-g)\left(1-\frac{a q}{h b}\right)\left(1-\frac{a q}{h c}\right)\left(1-\frac{a q}{h d}\right)\left(1-\frac{a q}{h e}\right)\left(1-\frac{a q}{h f}\right)}{\left(1-\frac{a q}{h}\right)\left(1-\frac{a q^{2}}{h}\right)} \Phi_{+}(h-) \\
-\frac{g\left(1-\frac{h}{g}\right)\left(1-\frac{a q}{b}\right)\left(1-\frac{a q}{c}\right)\left(1-\frac{a q}{d}\right)\left(1-\frac{a q}{e}\right)\left(1-\frac{a q}{f}\right)}{(1-a q)\left(1-a q^{2}\right)} \Phi=0 .
\end{gathered}
$$

Proof. First we indicate the proof for

$$
\Phi^{(b)}(a ; b, c, d, e, f, g, h ; q) .
$$

Assuming that (2.8) is valid, we apply Bailey's transformation (see Gasper and Rahman [7], (2.30), p. 56) to each of $\Phi^{(b)}+(g-), \Phi^{(b)}+(h-)$ and $\Phi^{(b)}$. Subsequently we have three pairs of ${ }_{10} \phi_{9}$ 's corresponding to the three $\Phi$ 's. We can pick out one ${ }_{10} \phi_{9}$ from each of the three pairs so that this set of three ${ }_{10} \phi_{9}$ 's and the remaining set of three both separately satisfy valid three-term contiguous relations. In fact, one set satisfies the relation we obtain by applying Lemma 2.1 to ${ }_{10} W_{9}\left(\frac{b h}{g} ; b, \frac{a q}{c g}, \frac{a q}{d g}, \frac{a q}{e g}, \frac{a q}{f g}, \frac{b h}{a}, h ; q\right)$. The other set satisfies the relation obtained by applying Lemma 2.1 to ${ }_{10} W_{9}\left(\frac{b g}{h} ; b, \frac{a q}{c h}, \frac{a q}{d h}, \frac{a q}{e h}, \frac{a q}{f h}, \frac{b h}{a}, g ; q\right)$. This completes the proof of (2.8) when ' $b$ ' is the distinguished parameter. We have a similar proof when ' $h$ ' is the distinguished parameter. This time one combination of three ${ }_{10} \phi_{9}$ 's gets disposed of exactly as in the previous case. The other combination of three ${ }_{10} \phi_{9}$ 's constitutes a relation which is the same as that obtained by applying Lemma 2.2 to the series

$$
{ }_{10} W_{9}\left(\frac{h g}{b} ; h, \frac{a q}{b c}, \frac{a q}{b d}, \frac{a q}{b e}, \frac{a q}{b f}, \frac{h g}{a q}, g q ; q\right) \text {. }
$$

This completes the proof of Theorem 2.3. 
Theorem 2.4. Irrespective of the choice of the distinguished parameter, $\Phi$ satisfies the relation

$$
\begin{aligned}
& \frac{g(1-h)\left(1-\frac{a}{h}\right)\left(1-\frac{a q}{h}\right)\left(1-\frac{a q}{g b}\right)\left(1-\frac{a q}{g c}\right)\left(1-\frac{a q}{g d}\right)\left(1-\frac{a q}{g e}\right)\left(1-\frac{a q}{g f}\right)}{\left(1-\frac{h q}{g}\right)} \\
& \quad \times[\Phi(g-, h+)-\Phi] \\
& \quad-\frac{h(1-g)\left(1-\frac{a}{g}\right)\left(1-\frac{a q}{g}\right)\left(1-\frac{a q}{h b}\right)\left(1-\frac{a q}{h c}\right)\left(1-\frac{a q}{h d}\right)\left(1-\frac{a q}{h e}\right)\left(1-\frac{a q}{h f}\right)}{\left(1-\frac{g q}{h}\right)} \\
& \quad \times[\Phi(h-, g+)-\Phi] \\
& \quad-\frac{a q}{h}\left(1-\frac{h}{g}\right)\left(1-\frac{g h}{a q}\right)(1-b)(1-c)(1-d)(1-e)(1-f) \Phi=0 .
\end{aligned}
$$

Proof. The result can be obtained from Theorem 2.3, if we substitute in (2.8) the value of $\Phi_{+}(g-)$ as given by $(2.3)$ and the value of $\Phi_{+}(h-)$ which would be obtained from a $g \leftrightarrow h$ interchange of $(2.3)$.

Theorem 2.5. Irrespective of the choice of the distinguished parameter, $\Phi$ satisfies the relation

$$
\begin{aligned}
& \frac{a g q}{h} \frac{(1-a q)\left(1-a q^{2}\right)\left(1-\frac{a q}{g b}\right)\left(1-\frac{a q}{g c}\right)\left(1-\frac{a q}{g d}\right)\left(1-\frac{a q}{g e}\right)\left(1-\frac{a q}{g f}\right)}{\left(1-\frac{a q}{g}\right)\left(1-\frac{a q^{2}}{g}\right)\left(1-\frac{a q}{b}\right)\left(1-\frac{a q}{c}\right)\left(1-\frac{a q}{d}\right)\left(1-\frac{a q}{e}\right)\left(1-\frac{a q}{f}\right)} \\
& \quad \times\left(1-\frac{g h}{a q}\right)(1-h)(1-b)(1-c)(1-d)(1-e)(1-f) \Phi_{+}(g-) \\
& \quad-\frac{q\left(1-\frac{a}{g}\right)\left(1-\frac{a q}{g}\right)\left(1-\frac{a}{h}\right)\left(1-\frac{a q}{h}\right)\left(1-\frac{a}{b}\right)\left(1-\frac{a}{c}\right)\left(1-\frac{a}{d}\right)\left(1-\frac{a}{e}\right)\left(1-\frac{a}{f}\right)}{\left(1-\frac{a}{q}\right)(1-a)} \Phi_{-}(g+) \\
& \quad-\left[\frac{a q}{h}\left(1-\frac{h}{g}\right)\left(1-\frac{g h}{a q}\right)(1-b)(1-c)(1-d)(1-e)(1-f)\right. \\
& \quad+\frac{g^{2} q^{2}}{h} \frac{\left(1-\frac{a q}{g}\right)\left(1-\frac{a}{h}\right)\left(1-\frac{a q}{h}\right)\left(1-\frac{h}{q}\right)\left(1-\frac{a}{g b}\right)\left(1-\frac{a}{g c}\right)\left(1-\frac{a}{g d}\right)\left(1-\frac{a}{g e}\right)\left(1-\frac{a}{g f}\right)}{\left(1-\frac{g q}{h}\right)\left(1-\frac{a}{g q}\right)} \\
& \left.\quad-\frac{h(1-g)\left(1-\frac{a}{g}\right)\left(1-\frac{a q}{g}\right)\left(1-\frac{a q}{h b}\right)\left(1-\frac{a q}{h c}\right)\left(1-\frac{a q}{h d}\right)\left(1-\frac{a q}{h e}\right)\left(1-\frac{a q}{h f}\right)}{\left(1-\frac{g q}{h}\right)}\right] \Phi=0 .
\end{aligned}
$$

Proof. First we make the parameter replacements

$$
(a, b, c, d, e, f, g, h) \rightarrow\left(\frac{a}{q^{2}}, \frac{b}{q}, \frac{c}{q}, \frac{d}{q}, \frac{e}{q}, \frac{f}{q}, g, \frac{h}{q}\right)
$$

in (2.8), which yields the value of $\Phi(g+, h-)$ in terms of $\Phi_{-}(g+)$ and $\Phi$. Also, Lemma 2.1' gives the value of $\Phi(g-, h+)$ in terms of $\Phi_{+}(g-)$ and $\Phi$. Substituting these values of $\Phi(g+, h-)$ and $\Phi(g-, h+)$ into (2.9) and simplifying, we obtain (2.10).

\section{Solutions to A DiffEREnCE EQUATION AND A CONTINUED FRACTION}

In the contiguous relation (2.9) of Theorem 2.4, we make the replacements

$$
(h, g) \rightarrow\left(h q^{-n}, \frac{s}{h} q^{n-1}\right),
$$


where, to account for the balance condition, we choose

$$
s=\frac{a^{3} q^{3}}{b c d e f} .
$$

After renormalization, the above relation can be reduced to the second order finite difference equation

$$
\begin{aligned}
& X_{n+1}-a_{n} X_{n}+b_{n} X_{n-1}=0, \\
& a_{n}=A_{n}+B_{n}+\frac{s q^{2 n-1}}{a h^{2}} \frac{\left(1-\frac{s}{a q^{2}}\right)(1-b)(1-c)(1-d)(1-e)(1-f)}{\left(1-\frac{a}{h} q^{n+1}\right)\left(1-\frac{s}{a h} q^{n-2}\right)}, \\
& b_{n}=A_{n-1} B_{n},
\end{aligned}
$$

where

$$
\begin{aligned}
A_{n}= & \frac{\left(1-\frac{s q^{n-1}}{h}\right)\left(1-\frac{s q^{n-1}}{a h}\right)\left(1-\frac{a q^{n+1}}{b h}\right)\left(1-\frac{a q^{n+1}}{c h}\right)}{\left(1-\frac{s q^{2 n}}{h^{2}}\right)\left(1-\frac{s q^{2 n-1}}{h^{2}}\right)} \\
& \times \frac{\left(1-\frac{a q^{n+1}}{d h}\right)\left(1-\frac{a q^{n+1}}{e h}\right)\left(1-\frac{a q^{n+1}}{f h}\right)}{\left(1-\frac{a q^{n+1}}{h}\right)} \\
B_{n}= & \frac{q\left(1-\frac{q^{n}}{h}\right)\left(1-\frac{a q^{n}}{h}\right)\left(1-\frac{b s q^{n-2}}{a h}\right)\left(1-\frac{c s q^{n-2}}{a h}\right)}{\left(1-\frac{s q^{2 n-1}}{h^{2}}\right)\left(1-\frac{s q^{2 n-2}}{h^{2}}\right)} \\
& \times \frac{\left(1-\frac{d s q^{n-2}}{a h}\right)\left(1-\frac{s q^{n-2}}{a h}\right)\left(1-\frac{f s q^{n-2}}{a h}\right)}{\left(1-\frac{s q^{n-2}}{a h}\right)} .
\end{aligned}
$$

It follows that one of the solutions of the finite difference equation (3.2) is

$$
\begin{aligned}
X_{n}^{(1)}= & \frac{\left(\frac{s q^{2 n-1}}{h^{2}}, \frac{a q^{n+1}}{h}\right)_{\infty}}{\left(\frac{s q^{n-1}}{h}, \frac{s q^{n-1}}{a h}, \frac{a q^{n+1}}{b h}, \frac{a q^{n+1}}{c h}, \frac{a q^{n+1}}{d h}, \frac{a q^{n+1}}{e h}, \frac{a q^{n+1}}{f h}\right)_{\infty}} \\
& \times \Phi\left(a ; b, c, d, e, f, \frac{s q^{n-1}}{h}, h q^{-n} ; q\right) .
\end{aligned}
$$

In fact, (3.3) gives not one solution but seven different pairwise linearly independent solutions, depending on the choice of the distinguished parameter out of the seven parameters $b, c, d, e, f, s q^{n-1} / h, h q^{-n}$ which define $\Phi$ (see (1.8)). In order to distinguish between these seven solutions we shall write $X_{n}^{(1), p}$ instead of $X_{n}^{(1)}, p$ being the distinguished parameter. Thus $X_{n}^{(1)}$ represents a set of seven solutions to equation (3.2).

A second set of seven pairwise linearly independent solutions to (3.2) is obtained by applying what we call a 'reflection transformation' to (3.2) and (3.3) [9], [10]. That is, in (3.2) we make the parameter replacements

$$
\left(a, b, c, d, e, f, s q^{n-1} / h, h q^{-n}\right) \rightarrow\left(q / a, q / b, q / c, q / d, q / e, q / f, h q^{-n+2} / s, q^{n+1} / h\right) .
$$

It can then be seen that [25]

$$
a_{n} \rightarrow a_{n} h^{2} q^{-2 n+1} / s, \quad b_{n} \rightarrow b_{n+1} h^{4} q^{-4 n} / s^{2} .
$$

It is easy to verify that $b_{n} \rightarrow b_{n+1} h^{4} q^{-4 n} / s^{2}$. To check $a_{n} \rightarrow a_{n} h^{2} q^{-2 n+1} / s$, we need to verify a polynomial identity of degree 14 , which we have done on a computer employing MAPLE software. 
Having made the above parameter replacements, we can renormalize so as to arrive back at the equation (3.2). Consequently, a second set of seven solutions is given by

$$
\begin{aligned}
X_{n}^{(2)}= & \frac{\left(\frac{s q^{2 n-1}}{h^{2}}, \frac{s q^{n}}{a h}\right)_{\infty}}{\left(\frac{q^{n+1}}{h}, \frac{a q^{n}}{h}, \frac{b s q^{n-1}}{a h}, \frac{c s q^{n-1}}{a h}, \frac{d s q^{n-1}}{a h}, \frac{e s q^{n-1}}{a h}, \frac{f s q^{n-1}}{a h}\right)_{\infty}} \\
& \times \Phi\left(q / a ; q / b, q / c, q / d, q / e, q / f, h q^{-n+2} / s, q^{n+1} / h ; q\right) .
\end{aligned}
$$

The seven solutions represented by the different $\Phi$ 's of (3.6) will be denoted by $X_{n}^{(2), p}$, where $p$ is chosen as the distinguished parameter out of the seven parameters $q / b, q / c, q / d, q / e, q / f, h q^{-n+2} / s, q^{n+1} / h$.

We now look for additional solutions to (3.2) which may be obtained by suitable parameter replacements. One solution which can be obtained with the help of the three-term contiguous relation (2.10) derived in Theorem 2.5 now follows.

First we interchange $g \leftrightarrow b$ in (2.10) and then make the substitutions

$$
\begin{aligned}
& a=\frac{S^{2} q^{2 n-2}}{A H^{2}}, \quad b=\frac{S}{A q}, \quad c=\frac{B S q^{n-1}}{A H}, \quad d=\frac{C S q^{n-1}}{A H}, \\
& e=\frac{D S}{A H} q^{n-1}, \quad f=\frac{E S}{A H} q^{n-1}, \quad g=\frac{F S q^{n-1}}{A H}, \quad h=\frac{S q^{n-1}}{H},
\end{aligned}
$$

ensuring that bcdefgh $=a^{3} q^{2}$ and $S=\frac{A^{3} q^{3}}{B C D E F}$.

Next we renormalize and get back to equation (3.2) with lower case letters $a, b$, $c, d, e, f, s$ replaced by capitals. Thus we arrive at a third set of solutions

$$
\begin{aligned}
& X_{n}^{(3)}=\left(\frac{s}{a q}\right)^{n} \frac{\left(\frac{s q^{n}}{a h}, \frac{s q^{2 n}}{h^{2}}, \frac{s q^{2 n-1}}{h^{2}}\right)_{\infty}}{\left(\frac{s q^{n-1}}{h}, \frac{q^{n+1}}{h}, \frac{s^{2} q^{2 n-1}}{a h^{2}}\right)_{\infty}} \\
& \times \frac{\left(\frac{s q^{n}}{b h}, \frac{s q^{n}}{c h}, \frac{s q^{n}}{d h}, \frac{s q^{n}}{e h}, \frac{s q^{n}}{f h}\right)_{\infty}}{\left(\frac{a q^{n+1}}{b h}, \frac{a q^{n+1}}{c h}, \frac{a q^{n+1}}{d h}, \frac{a q^{n+1}}{e h}, \frac{a q^{n+1}}{f h}\right)_{\infty}\left(\frac{b s q^{n-1}}{a h}, \frac{c s q^{n-1}}{a h}, \frac{d s q^{n-1}}{a h}, \frac{e s q^{n-1}}{a h}, \frac{f s q^{n-1}}{a h}\right)_{\infty}} \\
& \times \Phi\left(\frac{s^{2} q^{2 n-2}}{a h^{2}} ; \frac{s}{a q}, \frac{b s q^{n-1}}{a h}, \frac{c s q^{n-1}}{a h}, \frac{d s q^{n-1}}{a h}, \frac{e s q^{n-1}}{a h}, \frac{f s q^{n-1}}{a h}, \frac{s q^{n-1}}{h} ; q\right) .
\end{aligned}
$$

We might expect that (3.8) represents seven new solutions $X_{n}^{(3), p}$, where $p$ is chosen as the distinguished parameter out of the parameters $\frac{s}{a q}, \frac{b s q^{n-1}}{a h}, \frac{c s q^{n-1}}{a h}$, $\frac{d s q^{n-1}}{a h}, \frac{e s q^{n-1}}{a h}, \frac{f s q^{n-1}}{a h}$ and $\frac{s q^{n-1}}{h}$. However, we find that one of these seven solutions, viz., $X_{n}^{(3), \frac{s}{h} q^{n-1}}$, is simply a constant multiple of a solution of the first set, viz., $X_{n}^{(1), \frac{s}{h} q^{n-1}}$. The actual relation between the two solutions is

$$
X_{n}^{(1), \frac{s}{h} q^{n-1}}=\frac{(a q, b, c, d, e, f, h, q / h)_{\infty}}{(a q / b, a q / c, a q / d, a q / e, a q / f, s / a q, a h q / s, s / a h)_{\infty}} X_{n}^{(3), \frac{s}{h} q^{n-1}} .
$$

Thus (3.8) gives only six new pairwise linearly independent solutions $X_{n}^{(3), p}$, where $p$ is the distinguished parameter chosen out of $\frac{s}{a q}, \frac{b s q^{n-1}}{a h}, \frac{c s q^{n-1}}{a h}, \frac{d s q^{n-1}}{a h}, \frac{e s q^{n-1}}{a h}$, $\frac{f s q^{n-1}}{a h}$. 
We now apply the reflection transformation (3.4) to the solution $X_{n}^{(3)}$, which enables us to arrive at the solution

$$
\begin{aligned}
& X_{n}^{(4)}=\left(\frac{a q^{2}}{s}\right)^{n} \frac{\left(\frac{s^{2} q^{2 n-3}}{a h^{2}}\right)_{\infty}}{\left(\frac{s}{a h} q^{n-1}, \frac{s q^{n-1}}{b h}, \frac{s q^{n-1}}{c h}, \frac{s q^{n-1}}{d h}, \frac{s q^{n-1}}{e h}, \frac{s q^{n-1}}{f h}\right)_{\infty}} \\
& \times \Phi\left(\frac{a h^{2} q^{-2 n+3}}{s^{2}} ; \frac{a q^{2}}{s}, \frac{a h q^{-n+2}}{b s}, \frac{a h q^{-n+2}}{c s}, \frac{a h q^{-n+2}}{d s}, \frac{a h q^{-n+2}}{e s},\right. \\
&\left.\frac{a h q^{-n+2}}{f s}, \frac{h q^{-n+2}}{s} ; q\right) .
\end{aligned}
$$

This gives a set of six new solutions $X_{n}^{(4), p}$, where $p$ is chosen out of the six parameters $\frac{a q^{2}}{s}, \frac{a h q^{-n+2}}{b s}, \frac{a h q^{-n+2}}{c s}, \frac{a h q^{-n+2}}{d s}, \frac{a h q^{-n+2}}{e s}, \frac{a h q^{-n+2}}{f s}$. The remaining parameter does not give a new solution, since it is easily seen that $X_{n}^{(4), \frac{h}{s} q^{-n+2}}$ is a constant multiple of the already obtained solution $X_{n}^{(2), \frac{h}{s} q^{-n+2}}$.

A fifth set of solutions to (3.2) can be obtained as follows. After interchanging $g \leftrightarrow b$ in the contiguous relation (2.10) of Theorem 2.5, we make the substitutions

$$
\begin{aligned}
& a=\frac{A q^{2 n+1}}{H^{2}}, \quad b=\frac{A q^{2}}{S}, \quad c=\frac{A q^{n+1}}{B H}, \quad d=\frac{A q^{n+1}}{C H}, \\
& e=\frac{A q^{n+1}}{D H}, \quad f=\frac{A q^{n+1}}{E H}, \quad g=\frac{A q^{n+1}}{F H}, \quad h=\frac{q^{n+1}}{H},
\end{aligned}
$$

which again ensures that bcdefgh $=a^{3} q^{2}$ and $S=\frac{A^{3} q^{3}}{B C D E F}$. Renormalizing, we obtain (3.2) with lower case letters $a, b, c, d, e, f, s$ replaced by capital letters. This gives another set of solutions

$$
\begin{aligned}
& X_{n}^{(5)}=\left(\frac{a q^{2}}{s}\right)^{n} \frac{\left(\frac{s}{h^{2}} q^{2 n-1}\right)_{\infty}\left(\frac{s}{h^{2}} q^{2 n}\right)_{\infty}\left(\frac{a q^{n+1}}{h}\right)_{\infty}}{\left(\frac{a}{h^{2}} q^{2 n+2}\right)_{\infty}\left(\frac{s}{h} q^{n-1}\right)_{\infty}\left(\frac{q^{n+1}}{h}\right)_{\infty}} \\
& \times \frac{\left(\frac{b q^{n+1}}{h}, \frac{c q^{n+1}}{h}, \frac{d q^{n+1}}{h}, \frac{e q^{n+1}}{h}, \frac{f q^{n+1}}{h}\right)_{\infty}}{\left(\frac{b s}{a h} q^{n-1}, \frac{c s}{a h} q^{n-1}, \frac{d s}{a h} q^{n-1}, \frac{e s}{a h} q^{n-1}, \frac{f s}{a h} q^{n-1}\right)_{\infty}\left(\frac{a q^{n+1}}{b h}, \frac{a q^{n+1}}{c h}, \frac{a q^{n+1}}{d h}, \frac{a q^{n+1}}{e h}, \frac{a q^{n+1}}{f h}\right)_{\infty}} \\
& \times \Phi\left(\frac{a q^{2 n+1}}{h^{2}} ; \frac{a q^{2}}{s}, \frac{a q^{n+1}}{b h}, \frac{a q^{n+1}}{c h}, \frac{a q^{n+1}}{d h}, \frac{a q^{n+1}}{e h}, \frac{a q^{n+1}}{f h}, \frac{q^{n+1}}{h} ; q\right) .
\end{aligned}
$$

Depending on the choice of the distinguished parameter, there are seven solutions given by (3.12). However, two of these seven solutions, viz., $X_{n}^{(5), \frac{q^{n+1}}{h}}$, and $X_{n}^{(5), \frac{a q^{2}}{s}}$ are constant multiples of $X_{n}^{(2), \frac{q^{n+1}}{h}}$ and $X_{n}^{(4), \frac{a q^{2}}{s}}$, obtained before. Hence (3.12) gives five new solutions.

Next, we apply the reflection transformation to the solution $X_{n}^{(5)}$. This leads to the solution

$$
\begin{aligned}
X_{n}^{(6)} & =\left(\frac{s}{a q}\right)^{n} \frac{\left(\frac{a}{h^{2}} q^{2 n}\right)_{\infty}}{\left(\frac{a}{h} q^{n}\right)_{\infty}\left(\frac{b}{h} q^{n}, \frac{c}{h} q^{n}, \frac{d}{h} q^{n}, \frac{e}{h} q^{n}, \frac{f}{h} q^{n}\right)_{\infty}} \\
& \times \Phi\left(\frac{h^{2}}{a} q^{-2 n} ; \frac{s}{a q}, \frac{b h}{a} q^{-n}, \frac{c h}{a} q^{-n}, \frac{d h}{a} q^{-n}, \frac{e h}{a} q^{-n}, \frac{f h}{a} q^{-n}, h q^{-n} ; q\right) .
\end{aligned}
$$


Again, (3.13) represents only five new solutions, because two of the seven solutions, viz., $X_{n}^{(6), h q^{-n}}$ and $X_{n}^{(6), \frac{s}{a q}}$, are just constant multiples of the solutions $X_{n}^{(1), h q^{-n}}$ and $X_{n}^{(3), \frac{s}{a q}}$ respectively.

Finally, let us make the following parameter substitutions in the contiguous relation (2.9) of Theorem 2.4:

$$
\begin{aligned}
& a=\frac{B^{2}}{A}, \quad b=B, \quad c=\frac{B C}{A}, \quad d=\frac{B D}{A}, \\
& e=\frac{B E}{A}, \quad f=\frac{B F}{A}, \quad g=\frac{B S q^{n-1}}{A H}, \quad h=\frac{B H q^{-n}}{A} .
\end{aligned}
$$

Simplifying and renormalizing, we again arrive at (3.2) provided we can verify an identity in polynomials of degree 14. This also we have done with the help of MAPLE software. Consequently we obtain the solution

$$
\begin{aligned}
X_{n}^{(7)}= & \frac{\left(\frac{s}{h^{2}} q^{2 n-1}\right)_{\infty}\left(\frac{b}{h} q^{n+1}\right)_{\infty}}{\left(\frac{b s}{a h} q^{n-1}, \frac{s}{b h} q^{n-1}, \frac{q^{n+1}}{h}, \frac{a q^{n+1}}{c h}, \frac{a q^{n+1}}{d h}, \frac{a q^{n+1}}{e h}, \frac{a q^{n+1}}{f h}\right)_{\infty}} \\
& \times \Phi\left(\frac{b^{2}}{a} ; b, \frac{b c}{a}, \frac{b d}{a}, \frac{b e}{a}, \frac{b f}{a}, \frac{b s q^{n-1}}{a h}, \frac{b h q^{-n}}{a} ; q\right) .
\end{aligned}
$$

The solutions $X_{n}^{(7), b}, X_{n}^{(7), \frac{b s}{a h} q^{n-1}}, X_{n}^{(7), \frac{b h q-n}{a}}$ are clearly constant multiples of solutions obtained before. Hence $X_{n}^{(7)}$ represents a set of four new solutions.

Using reflection on $X_{n}^{(7)}$, we obtain the solution

$$
\begin{aligned}
X_{n}^{(8)}= & \frac{\left(\frac{s}{h^{2}} q^{2 n}, \frac{s}{b h} q^{n}\right)_{\infty}}{\left(\frac{s}{h} q^{n-1}, \frac{a q^{n+1}}{b h}, \frac{b q^{n}}{h}\right)_{\infty}\left(\frac{c s}{a h} q^{n-1}, \frac{d s}{a h} q^{n-1}, \frac{e s}{a h} q^{n-1}, \frac{f s}{a h} q^{n-1}\right)_{\infty}} \\
& \times \Phi\left(\frac{a q}{b^{2}} ; \frac{q}{b}, \frac{a q}{b c}, \frac{a q}{b d}, \frac{a q}{b e}, \frac{a q}{b f}, \frac{a h q^{-n+2}}{b s}, \frac{a q^{n+1}}{b h} ; q\right) .
\end{aligned}
$$

$X_{n}^{(8)}$ gives only four additional solutions, because three of the seven solutions, viz., $X_{n}^{(8), \frac{q}{b}}, X_{n}^{(8), \frac{a h}{b s} q^{-n+2}}$ and $X_{n}^{(8), \frac{a q^{n+1}}{b h}}$, are constant multiples of previously obtained solutions.

Parameter interchanges $b \leftrightarrow(c, d, e, f)$ in solutions $X_{n}^{(7)}$ and $X_{n}^{(8)}$ give us additional solutions. We find that there are twelve more solutions obtained in this manner, the rest being constant multiples of previous solutions.

Combining (3.3), (3.6), (3.8), (3.10), (3.12), (3.13), (3.15), and (3.16), we have fifty-six pairwise linearly independent solutions to the three-term recurrence (3.2). Any three of these fifty-six solutions are connected by a three-term transformation formula. We shall derive such a connection formula in $\S 6$.

For the special case $h=1$ and $s=q, q^{2}, \ldots$, the solutions $X_{n}^{(1)}$ and $X_{n}^{(2)}$ were obtained in [10]. Writing $h=1$ and $s=q, q^{2}, \ldots$ in (3.2), (3.3) and (3.6), we get respectively $[10,(2.6),(2.9),(2.12)]$ but with a different normalization.

Large $n$ asymptotics of the solutions and a continued fraction. Since $|q|<$ 1 , we have from $(3.2)$

$$
\lim _{n \rightarrow \infty} a_{n}=1+q, \quad \lim _{n \rightarrow \infty} b_{n}=q .
$$


It follows that (3.2) has solutions whose large $n$ asymptotics is either

$$
X_{n} \stackrel{n \rightarrow \infty}{\approx} \text { const. }
$$

or

$$
X_{n} \stackrel{n \rightarrow \infty}{\approx} \text { const. } q^{n}
$$

The latter characterizes the minimal solution to (3.2). We therefore proceed to examine the asymptotics of the solutions obtained above. From (3.3) we have in a straightforward manner

$$
\begin{aligned}
& X_{n}^{(1), b} \stackrel{n \rightarrow \infty}{\approx}_{8} W_{7}\left(a ; b, c, d, e, f ; \frac{s}{a q}\right) \\
& +\frac{\left(a q, c, d, e, f, \frac{b}{a}, \frac{b q}{c}, \frac{b q}{d}, \frac{b q}{e}, \frac{b q}{f}, h, \frac{q}{h}, \frac{b h q^{2}}{s}, \frac{s}{b h q}\right)_{\infty}}{\left(\frac{b^{2} q}{a}, \frac{b c}{a}, \frac{b d}{a}, \frac{b e}{a}, \frac{b f}{a}, \frac{a}{b}, \frac{a q}{c}, \frac{a q}{d}, \frac{a q}{e}, \frac{a q}{f}, \frac{b h}{a}, \frac{a q}{b h}, \frac{a h q^{2}}{s}, \frac{s}{a h q}\right)_{\infty}} \\
& \times{ }_{8} W_{7}\left(\frac{b^{2}}{a} ; b, \frac{b c}{a}, \frac{b d}{a}, \frac{b e}{a}, \frac{b f}{a} ; \frac{s}{a q}\right), \quad\left|\frac{s}{a q}\right|<1 .
\end{aligned}
$$

We will have analogous results with $c, d, e$ or $f$ as distinguished parameter. We also easily have

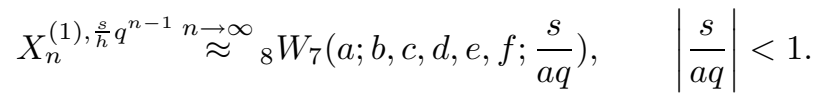

In order to calculate the asymptotics of $X_{n}^{(1), h q^{-n}}$, we first apply the transformation [7, (2.30), p. 56] to the $\Phi^{\left(h q^{-n}\right)}$ in (3.3). While working out the asymptotics of the two resulting ${ }_{10} \phi_{9}$ series, some care is necessary, since when $n$ is large, the terms near two parts of the series are important (see Bailey [4], p. 128). Thus it is convenient to break each ${ }_{10} \phi_{9}$ series into three parts

$$
\sum_{k=0}^{n / 2}+\sum_{k=n / 2}^{3 n / 2}+\sum_{k=3 n / 2}^{\infty},
$$

the third sum tending to 0 as $n \rightarrow \infty$. The first summation gives a ${ }_{4} \phi_{3}$ while the second gives a ${ }_{4} \psi_{4}$. We obtain

$$
\begin{aligned}
& X_{n}^{(1), h q^{-n}} \stackrel{n \rightarrow \infty}{\approx} \frac{\left(a q, c, \frac{a q}{d b}, \frac{a q}{e b}, \frac{a q}{f b}, \frac{s b}{a q}, \frac{h}{a}, \frac{h q}{c}, \frac{a h q^{2}}{b s}, \frac{b d h}{a}, \frac{b e h}{a}, \frac{b f h}{a}\right)_{\infty}}{\left(\frac{c}{b}, \frac{a q}{b}, \frac{a q}{d}, \frac{a q}{e}, \frac{a q}{f}, \frac{s}{a q}, \frac{b h q}{c}, \frac{b h}{a}, \frac{a h q^{2}}{s}, \frac{d h}{a}, \frac{e h}{a}, \frac{f h}{a}\right)_{\infty}} \\
& \times \frac{\left(\frac{a q}{h}, \frac{c}{h}, \frac{b s}{a h q}, \frac{a q}{b d h}, \frac{a q}{b e h}, \frac{a q}{b f h}\right)_{\infty}}{\left(\frac{c}{b h}, \frac{a q}{b h}, \frac{s}{a h q}, \frac{a q}{d h}, \frac{a q}{e h}, \frac{a q}{f h}\right)_{\infty}}\left[4 \phi_{3}\left(\begin{array}{c}
\frac{a q}{c d}, \frac{a q}{c e}, \frac{a q}{c f}, b \\
\left.\frac{b q}{c}, \frac{b s}{a q}, \frac{a q}{c} ; q\right)
\end{array}\right)\right. \\
& -\frac{c}{b h} \frac{\left(\frac{c q}{b h}, \frac{q}{h}, \frac{c s}{a h q}, \frac{a q}{b h}, \frac{a q}{d c}, \frac{a q}{e c}, \frac{a q}{f c}, b\right)_{\infty}}{\left(\frac{a q}{b d h}, \frac{a q}{b e h}, \frac{a q}{b f h}, \frac{c}{h}, \frac{b q}{c}, \frac{b s}{a q}, \frac{a q}{c}, q\right)_{\infty}}{ }_{4} \psi_{4}\left(\begin{array}{c}
\frac{b h}{c}, h, \frac{a h q^{2}}{c s}, \frac{b h}{a} \\
\left.\left.\frac{b d h}{a}, \frac{b e h}{a}, \frac{b f h}{a}, \frac{h q}{c} ; q\right)\right]
\end{array}\right. \\
& + \text { idem }(b ; c) \text {. }
\end{aligned}
$$


The asymptotics of the seven solutions represented by $X_{n}^{(1)}$ is thus completely given by (3.19), (3.20) and (3.21). There are similar results for $X_{n}^{(2)}$ solutions. We easily have

$$
\begin{aligned}
& X_{n}^{(2), \frac{q}{b}} \underset{{ }_{2} \rightarrow \infty}{\approx}\left[{ }_{8} W_{7}\left(\frac{q}{a} ; \frac{q}{b}, \frac{q}{c}, \frac{q}{d}, \frac{q}{e}, \frac{q}{f} ; \frac{a q^{2}}{s}\right)\right. \\
& +\frac{\left(\frac{q^{2}}{a}, \frac{a}{b}, \frac{q}{c}, \frac{q}{d}, \frac{q}{e}, \frac{q}{f}, \frac{c q}{b}, \frac{d q}{b}, \frac{e q}{b}, \frac{f q}{b}, \frac{h q^{2}}{s}, \frac{s}{h q}, \frac{h q}{b}, \frac{b}{h}\right)_{\infty}}{\left(\frac{a q^{2}}{b^{2}}, \frac{b}{a}, \frac{c q}{a}, \frac{d q}{a}, \frac{e q}{a}, \frac{f q}{a}, \frac{a q}{b c}, \frac{a q}{b d}, \frac{a q}{b e}, \frac{a q}{b f}, \frac{h q}{a}, \frac{a}{h}, \frac{a h q^{2}}{b s}, \frac{b s}{a h q}\right)_{\infty}} \\
& \left.\times{ }_{8} W_{7}\left(\frac{a q}{b^{2}} ; \frac{q}{b}, \frac{a q}{b c}, \frac{a q}{b d}, \frac{a q}{b e}, \frac{a q}{b f} ; \frac{a q^{2}}{s}\right)\right],\left|\frac{a q^{2}}{s}\right|<1,
\end{aligned}
$$

and

$$
X_{n}^{(2), \frac{q^{n+1}}{h}} \stackrel{{ }_{0} \infty}{{ }_{8} W_{7}}\left(\frac{q}{a} ; \frac{q}{b}, \frac{q}{c}, \frac{q}{d}, \frac{q}{e}, \frac{q}{f} ; \frac{a q^{2}}{s}\right), \quad\left|\frac{a q^{2}}{s}\right|<1
$$

The asymptotics of $X_{n}^{(2), \frac{h}{s} q^{-n+2}}$ is worked out exactly as we have done for $X_{n}^{(1), h q^{-n}}$. The result, being cumbersome, is not being given here.

For $X_{n}^{(3)}, X_{n}^{(4)}$ we have the following results which take care of all the related solutions:

$$
\begin{gathered}
X_{n}^{(3), \frac{b s}{a h} q^{n-1}} \underset{n \rightarrow \infty}{\approx} \frac{\left(\frac{s}{a q}, \frac{b q}{c}, \frac{b q}{d}, \frac{b q}{e}, \frac{b q}{f}, \frac{b q}{a}, \frac{b h q}{s}, \frac{s}{b h}\right)_{\infty}}{\left(\frac{b^{2} q}{a}, \frac{b c}{a}, \frac{b d}{a}, \frac{b e}{a}, \frac{b f}{a}, b, \frac{b h}{a}, \frac{a q}{b h}\right)_{\infty}} \\
\times{ }_{8} W_{7}\left(\frac{b^{2}}{a} ; \frac{b c}{a}, \frac{b d}{a}, \frac{b e}{a}, \frac{b f}{a}, b ; \frac{s}{a q}\right),\left|\frac{s}{a q}\right|<1, \\
X_{n}^{(3), \frac{s}{a q}} \underset{\approx}{\approx} \frac{\left(\frac{a}{b}, \frac{q}{c}, \frac{q}{d}, \frac{q}{e}, \frac{b s}{a q}, \frac{c s}{a q}, \frac{d s}{a q}, \frac{e s}{a q}, \frac{h^{2} q}{s}, \frac{s}{h^{2}}, \frac{h q}{f}, \frac{f}{h}\right)_{\infty}}{\left(\frac{s}{f}, \frac{f}{a}, h, \frac{q}{h}, \frac{b h}{a}, \frac{a q}{b h}, \frac{c h}{a}, \frac{a q}{c h}, \frac{d h}{a}, \frac{a q}{d h}, \frac{e h}{a}, \frac{a q}{e h}\right)_{\infty}} \\
\times{ }_{8} W_{7}\left(\frac{s}{f q} ; \frac{s}{a q}, \frac{a q}{b f}, \frac{a q}{c f}, \frac{a q}{d f}, \frac{a q}{e f} ; f\right) \\
+\frac{\left(\frac{a q}{b f}, \frac{a q}{c f}, \frac{a q}{d f}, \frac{a q}{e f}, \frac{b f s}{a^{2} q}, \frac{c f s}{a^{2} q}, \frac{d f s}{a^{2} q}, \frac{e f s}{a^{2} q}, \frac{h^{2} q}{s}, \frac{s}{h^{2}}, \frac{h q}{a}, \frac{a}{h}\right)_{\infty}}{\left(\frac{f s}{a^{2}}, \frac{a}{f}, \frac{b h}{a}, \frac{a q}{b h}, \frac{c h}{a}, \frac{a q}{c h}, \frac{d h}{a}, \frac{a q}{d h}, \frac{e h}{a}, \frac{a q}{e h}, \frac{f h}{a}, \frac{a q}{f h}\right)_{\infty}} \\
\times{ }_{8} W_{7}\left(\frac{s f}{a^{2} q} ; \frac{s}{a q}, \frac{q}{b}, \frac{q}{c}, \frac{q}{d}, \frac{q}{e} ; f\right), \\
|f|<1 .
\end{gathered}
$$

In order to obtain asymptotics of $X_{n}^{(4), \frac{a q^{2}}{s}}$, we first apply the transformation $([7]$, (2.30), p. 56) to the $\Phi$ in the solution and then work out the asymptotics. We get 


$$
\begin{aligned}
& X_{n}^{(4), \frac{a q^{2}}{s}} \\
& \underset{n \rightarrow \infty}{\approx}\left[\frac{\left(b, c, d, e, \frac{a q^{3}}{b s}, \frac{a q^{3}}{c s}, \frac{a q^{3}}{d s}, \frac{a q^{3}}{e s}, \frac{a h^{2} q^{4}}{s^{2}}, \frac{s^{2}}{a h^{2} q^{3}}, \frac{a h q^{2}}{f s}, \frac{f s}{a h q}\right)_{\infty}}{\left(\frac{a}{f}, \frac{f q^{3}}{s}, \frac{a h q^{2}}{s}, \frac{b h q^{2}}{s}, \frac{c h q^{2}}{s}, \frac{d h q^{2}}{s}, \frac{e h q^{2}}{s}, \frac{s}{a h q}, \frac{s}{b h q}, \frac{s}{c h q}, \frac{s}{d h q}, \frac{s}{e h q}\right)_{\infty}}\right. \\
& \times{ }_{8} W_{7}\left(\frac{f q^{2}}{s} ; \frac{a q^{2}}{s}, \frac{b f}{a}, \frac{c f}{a}, \frac{d f}{a}, \frac{e f}{a} ; \frac{q}{f}\right) \\
& +\frac{\left(\frac{b f}{a}, \frac{c f}{a}, \frac{d f}{a}, \frac{e f}{a}, \frac{a^{3} q^{3}}{b f s}, \frac{a^{3} q^{3}}{c f s}, \frac{a^{3} q^{3}}{d f s}, \frac{a^{3} q^{3}}{e f s}, \frac{a h^{2} q^{4}}{s^{2}}, \frac{s^{2}}{a h^{2} q^{3}}, \frac{h q^{2}}{s}, \frac{s}{h q}\right)_{\infty}}{\left(\frac{a^{2} q^{3}}{f s}, \frac{f}{a}, \frac{h b q^{2}}{s}, \frac{h c q^{2}}{s}, \frac{h d q^{2}}{s}, \frac{h e q^{2}}{s}, \frac{h f q^{2}}{s}, \frac{s}{h b q}, \frac{s}{h c q}, \frac{s}{h d q}, \frac{s}{h e q}, \frac{s}{h f q}\right)_{\infty}} \\
& \left.\times{ }_{8} W_{7}\left(\frac{a^{2} q^{2}}{f s} ; \frac{a q^{2}}{s}, b, c, d, e ; \frac{q}{f}\right)\right], \quad\left|\frac{q}{f}\right|<1 .
\end{aligned}
$$

The calculation of asymptotics of $X_{n}^{(4), \frac{a h q^{-n+2}}{b s}}$ is similar to that of $X_{n}^{(1), h q^{-n}}$.

Coming to solutions $X_{n}^{(5)}$ and $X_{n}^{(6)}$, we find that

$$
\begin{aligned}
& X_{n}^{(5), \frac{a q^{n+1}}{b h}} n \underset{\approx}{\approx} \frac{\left(\frac{a q^{2}}{s}, \frac{c q}{b}, \frac{d q}{b}, \frac{e q}{b}, \frac{f q}{b}, \frac{a q}{b}, \frac{h}{b}, \frac{b q}{h}\right)_{\infty}}{\left(\frac{a q^{2}}{b^{2}}, \frac{a q}{b c}, \frac{a q}{b d}, \frac{a q}{b e}, \frac{a q}{b f}, \frac{q}{b}, \frac{a h q^{2}}{b s}, \frac{b s}{a h q}\right)_{\infty}} \\
& \times{ }_{8} W_{7}\left(\frac{a q}{b^{2}} ; \frac{a q}{b c}, \frac{a q}{b d}, \frac{a q}{b e}, \frac{a q}{b f}, \frac{q}{b} ; \frac{a q^{2}}{s}\right), \quad\left|\frac{a q^{2}}{s}\right|<1,
\end{aligned}
$$

while $X_{n}^{(6), \frac{b h}{a} q^{-n}}$ is worked out as $X_{n}^{(1), h q^{-n}}$ and produces a similar expression giving constant asymptotics. The remaining solutions are structurally similar to the solutions for which we have calculated the asymptotics above. Thus we find that all the fifty-six solutions yield constant asymptotics as $n \rightarrow \infty$.

In order to obtain a minimal solution to (3.2) we make use of (3.20) and (3.23). We write

$$
W_{1}:={ }_{8} W_{7}\left(a ; b, c, d, e, f ; \frac{s}{a q}\right), \quad\left|\frac{s}{a q}\right|<1,
$$

and its analytic continuation otherwise, and

$$
W_{2}:={ }_{8} W_{7}\left(\frac{q}{a} ; \frac{q}{b}, \frac{q}{c}, \frac{q}{d}, \frac{q}{e}, \frac{q}{f} ; \frac{a q^{2}}{s}\right), \quad\left|\frac{a q^{2}}{s}\right|<1,
$$

and its analytic continuation otherwise. Define

$$
X_{n}^{(\min )}:=W_{2} X_{n}^{(1), \frac{s}{h} q^{n-1}}-W_{1} X_{n}^{(2), \frac{q^{n+1}}{h}} \stackrel{n \rightarrow \infty}{\approx} \text { const. } q^{n}
$$

from (3.20) and (3.23). The actual value of the constant in (3.30) will be given in section 7. It follows from (3.30) and (3.20) that

$$
\lim _{n \rightarrow \infty} \frac{X_{n}^{(\min )}}{X_{n}^{(1), \frac{s}{h} q^{n-1}}}=0, \quad\left|\frac{s}{q}\right|<|a|<\left|\frac{s}{q^{2}}\right| .
$$

Thus $X_{n}^{(\min )}$ given by (3.30) is a minimal solution of (3.2). An application of Pincherle's theorem [8], [19] then leads to the following result. 
Theorem 3.1. For $a_{n}, b_{n}$ defined by (3.2) the following continued fraction representation holds true:

$$
\begin{aligned}
& \frac{1}{a_{0}}-\frac{b_{1}}{a_{1}}-\frac{b_{2}}{a_{2}}-\ldots=\frac{W_{2} X_{0}^{(1), \frac{s}{h q}}-W_{1} X_{0}^{(2), \frac{q}{h}}}{b_{0}\left(W_{2} X_{-1}^{(1), \frac{s}{h q^{2}}}-W_{1} X_{-1}^{(2), \frac{1}{h}}\right)} \\
& =\frac{\left(1-\frac{s}{h^{2} q}\right)\left(1-\frac{s}{h^{2} q^{2}}\right)}{q} \\
& \times\left[W_{2} \frac{\left(\frac{a q}{h}\right)_{\infty}}{\left(\frac{s}{h q}, \frac{s}{a h q}, \frac{a q}{b h}, \frac{a q}{c h}, \frac{a q}{d h}, \frac{a q}{e h}, \frac{a q}{f h}\right)_{\infty}} \Phi^{\left(\frac{s}{h q}\right)}\left(a ; b, c, d, e, f, \frac{s}{h q}, h ; q\right)\right. \\
& \left.-W_{1} \frac{\left(\frac{s}{a h}\right)_{\infty}}{\left(\frac{q}{h}, \frac{a}{h}, \frac{b s}{a h q}, \frac{c s}{a h q}, \frac{d s}{a h q}, \frac{e s}{a h q}, \frac{f s}{a h q}\right)_{\infty}} \Phi^{\left(\frac{q}{h}\right)}\left(\frac{q}{a} ; \frac{q}{b}, \frac{q}{c}, \frac{q}{d}, \frac{q}{e}, \frac{q}{f}, \frac{h q^{2}}{s}, \frac{q}{h} ; q\right)\right] \\
& /\left[W_{2} \frac{\left(1-\frac{1}{h}\right)\left(1-\frac{b s}{a h q^{2}}\right)\left(1-\frac{c s}{a h q^{2}}\right)\left(1-\frac{d s}{a h q^{2}}\right)\left(1-\frac{e s}{a h q^{2}}\right)\left(1-\frac{f s}{a h q^{2}}\right)\left(\frac{a}{h}\right)_{\infty}}{\left(\frac{s}{h q}, \frac{s}{a h q^{2}}, \frac{a q}{b h}, \frac{a q}{c h}, \frac{a q}{d h}, \frac{a q}{e h}, \frac{a q}{f h}\right)_{\infty}}\right. \\
& \times \Phi^{\left(\frac{s}{h q^{2}}\right)}\left(a ; b, c, d, e, f, \frac{s}{h q^{2}}, h q ; q\right) \\
& -W_{1} \frac{\left(1-\frac{s}{h q^{2}}\right)\left(1-\frac{a}{b h}\right)\left(1-\frac{a}{c h}\right)\left(1-\frac{a}{d h}\right)\left(1-\frac{a}{e h}\right)\left(1-\frac{a}{f h}\right)\left(\frac{s}{a h q}\right)_{\infty}}{\left(\frac{q}{h}, \frac{a}{q h}, \frac{b s}{a q h}, \frac{c s}{a q h}, \frac{d s}{a q h}, \frac{e s}{a q h}, \frac{f s}{a q h}\right)_{\infty}} \\
& \left.\times \Phi^{\left(\frac{1}{h}\right)}\left(\frac{q}{a} ; \frac{q}{b}, \frac{q}{c}, \frac{q}{d}, \frac{q}{e}, \frac{q}{f}, \frac{h q^{3}}{s}, \frac{1}{h} ; q\right)\right] .
\end{aligned}
$$

For the special case $h=1$ we have

Corollary 3.2. If $a_{n}, b_{n}$ are defined by (3.2) with $h=1$, then the following continued fraction representation holds true (Masson [25]):

$$
\begin{aligned}
& \frac{1}{a_{0}}-\frac{b_{1}}{a_{1}}-\frac{b_{2}}{a_{2}}-\cdots \\
& =\frac{\left(1-\frac{s}{q}\right)\left(1-\frac{a}{q}\right)}{q\left(1-\frac{s}{a q}\right)\left(1-\frac{a}{b}\right)\left(1-\frac{a}{c}\right) \cdots\left(1-\frac{a}{f}\right)} \\
& \quad \times\left[10 W_{9}\left(\frac{q}{a} ; q, \frac{q^{2}}{s}, \frac{q}{b}, \cdots, \frac{q}{f} ; q\right)+\Pi_{110} W_{9}\left(a q ; q, \frac{a q^{2}}{s}, \frac{a q}{b}, \cdots, \frac{a q}{f} ; q\right)\right. \\
& \left.\quad-\frac{\left(q, a, a q, \frac{b s}{a q}, \cdots, \frac{f s}{a q}\right)_{\infty}}{\left(\frac{s}{q}, \frac{s}{a}, \frac{s}{a q}, \frac{a q}{b}, \cdots, \frac{a q}{f}\right)_{\infty}} \frac{W_{2}}{W_{1}}\right] /\left(1+\Pi_{0}\right),
\end{aligned}
$$

where

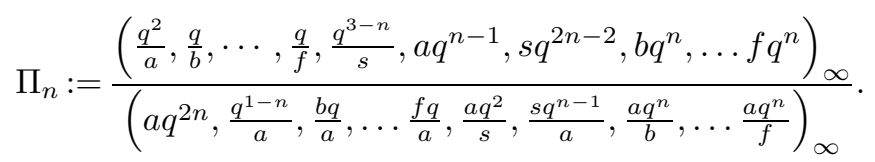

Proof. We take the limit of (3.32) as $h \rightarrow 1$. 
A special case of Corollary 3.2 for $s=q^{3}, q^{4}, \ldots$ was obtained in [10, (5.1), p. 438].

In the terminating case of Corollary 3.2 we have

Corollary 3.3. If $h=1$ and one of $\frac{a q}{b}, \frac{a q}{c}, \frac{a q}{d}, \frac{a q}{e}, \frac{a q}{f}=q^{-N}, N=0,1, \ldots$, then

$$
\begin{aligned}
& \frac{1}{a_{0}}-\frac{b_{1}}{a_{1}}-\frac{b_{2}}{a_{2}}-\cdots-\frac{b_{N}}{a_{N}} \\
& =\frac{a q}{s} \frac{(1-a q)}{(1-b)(1-c)(1-d)(1-e)(1-f)}{ }_{10} W_{9}\left(a q ; q, \frac{a q^{2}}{s}, \frac{a q}{b}, \ldots \frac{a q}{f} ; q\right) .
\end{aligned}
$$

Proof. We take the limit of (3.33) as, say, $\frac{a q}{f} \rightarrow q^{-N}$ (see Masson [25]).

Special cases of Corollary 3.3 for $s=q, q^{2}$ were given in [10]. The case $s=q^{2}$ may be reexpressed as Watson's $q$-analogue of Ramanujan's Entry 40 [10], [29]. To rederive the expressions in [10], note that for $s=q$ the ${ }_{10} W_{9}$ in (3.34) reduces to an ${ }_{8} W_{7}$ which is summable using Jackson's formula [7, (II.22), p. 238]. For $s=q^{2}$ the ${ }_{10} W_{9}$ reduces to a pair of summable ${ }_{8} W_{7}$ 's after using the contiguous relation (2.2).

It is also this last corollary which yields the rational biorthogonality discussed in Section 4. This is partly because the singularities in (3.34) are explicit. For example, there is clearly a pole singularity at $b=1$. The corresponding residue is explicit, since at $b=1$ the ${ }_{10} W_{9}$ again becomes a summable ${ }_{8} W_{7}$. Something similar happens when $b=q^{-k}, k=1,2, \cdots, N$ (see the calculation below from (4.13) to (4.14)).

\section{Discrete Rational Biorthogonality}

The continued fraction (3.34) can be put into the form of an $R_{I I}$-fraction [17]. Associated with this $R_{I I}$-fraction there is an explicit system of biorthogonal rational functions [30], [26], [25]. In this section we derive such a biorthogonal system using the methods in [17]. This gives the top level of an Askey type scheme of discrete rational biorthogonality for which we also outline five different next level limits and a $q \rightarrow 1$ limit. For the Askey-scheme of hypergeometric orthogonal polynomials, see $[20]$.

4 a). The top ${ }_{10} \phi_{9}$ level. In the three-term recurrence (3.2) we make the replacements

$$
b \rightarrow b e^{-\xi}, \quad c \rightarrow \mu b e^{\xi}, \quad h \rightarrow 1,
$$

with

$$
x:=\left(e^{\xi}+e^{-\xi} / \mu\right) / 2 .
$$

The coefficients $a_{n}$ and $b_{n}$ in (3.2) then become linear and quadratic functions of $x$ respectively, and the recurrence takes the form

$$
X_{n+1}(x)-\left(u_{n+1} x+v_{n+1}\right) X_{n}(x)+\gamma_{n}\left(x-\alpha_{n+1}\right)\left(x-\beta_{n+1}\right) X_{n-1}(x)=0 .
$$

After a renormalization this becomes the recurrence

$$
Y_{n+1}(x)-\left(x-c_{n+1}\right) Y_{n}(x)+\lambda_{n+1}\left(x-\alpha_{n+1}\right)\left(x-\beta_{n+1}\right) Y_{n-1}(x)=0
$$

associated with the $R_{I I}$-fraction

$$
R_{I I}(x)=\frac{1}{x-c_{1}}-\frac{\lambda_{2}\left(x-\alpha_{2}\right)\left(x-\beta_{2}\right)}{x-c_{2}}-\frac{\lambda_{3}\left(x-\alpha_{3}\right)\left(x-\beta_{3}\right)}{x-c_{3}}-\ldots .
$$


The connection between (4.3) and $\left(4.3^{\prime}\right)$ is given by

$$
c_{n+1}=-\frac{v_{n+1}}{u_{n+1}}, \quad \lambda_{n+1}=\frac{\gamma_{n}}{u_{n+1} u_{n}}, \quad Y_{n}=\frac{X_{n}}{\prod_{k=-1}^{n} u_{k}},
$$

but the renormalization factor $\prod_{k=-1}^{n} u_{k}$ will not enter our final formulas. We may explicitly calculate $c_{n+1}, \lambda_{n+1}, \alpha_{n+1}$ and $\beta_{n+1}$ from the original $a_{n}$ and $b_{n}$, but for our purposes we only note that

$$
\begin{aligned}
\gamma_{n}= & 4 \mu s q^{2 n-2}\left(1-q^{n}\right)\left(1-s q^{n-2}\right)\left(1-\frac{a}{d} q^{n}\right)\left(1-\frac{a}{e} q^{n}\right)\left(1-\frac{a}{f} q^{n}\right) \\
& \times\left(1-\frac{d s}{a} q^{n-2}\right)\left(1-\frac{e s}{a} q^{n-2}\right)\left(1-\frac{f s}{a} q^{n-2}\right), \\
s= & \frac{a^{3} q^{3}}{\mu b^{2} d e f},
\end{aligned}
$$

and the 'interpolation points' $\alpha_{n+1}, \beta_{n+1}$ are given by

$$
\begin{aligned}
\alpha_{n+1} & =\left(\frac{b s}{a} q^{n-2}+\frac{a}{b s \mu} q^{2-n}\right) / 2, \\
\beta_{n+1} & =\left(\frac{a}{b \mu} q^{n}+\frac{b}{a} q^{-n}\right) / 2 .
\end{aligned}
$$

In order to have explicit singularities in the continued fraction (4.4), we will need termination. For this we now choose

$$
f=a q^{N+1}, \quad N=0,1, \ldots,
$$

so that $\lambda_{N+2}=0$. We can then use the result of Corollary 3.4 with the replacement (4.1) to obtain

$$
\begin{aligned}
R_{I I}(x)= & \frac{u_{0} \mu b^{2} d e q^{N}(1-a q)}{a\left(1-b e^{-\xi}\right)\left(1-\mu b e^{\xi}\right)(1-d)(1-e)\left(1-a q^{N+1}\right)} \\
& \quad \times{ }_{10} W_{9}\left(a q ; q, \frac{a q e^{\xi}}{b}, \frac{a q e^{-\xi}}{\mu b}, \frac{a q}{d}, \frac{a q}{e}, \frac{\mu b^{2} d e q^{N}}{a}, q^{-N} ; q\right) \\
= & \sum_{k=0}^{N} \frac{R_{k}}{x-x_{k}}, \quad x=\frac{\left(e^{\xi}+\mu^{-1} e^{-\xi}\right)}{2} .
\end{aligned}
$$

An explicit polynomial solution to (4.3) is given by (3.3) with the replacement (4.1) and identification (4.2). Consequently the explicit polynomial solution of the first kind to $\left(4.3^{\prime}\right)$ is given by

$$
P_{n}(x)=\frac{\left(\frac{s}{q}, \frac{s}{a q}, \frac{a q e^{\xi}}{b}, \frac{a q e^{-\xi}}{\mu b}, \frac{a q}{d}, \frac{a q}{e}, q^{-N}\right)_{n} U_{n}(x)}{(a q)_{n}\left(\frac{s}{q}\right)_{2 n} \prod_{k=-1}^{n} u_{k}}
$$

where

$$
\begin{aligned}
U_{n}(x) & ={ }_{10} W_{9}\left(a ; b e^{-\xi}, \mu b e^{\xi}, d, e, a q^{N+1}, s q^{n-1}, q^{-n} ; q\right) \\
s & =\frac{a^{2} q^{2-N}}{\mu b^{2} d e} .
\end{aligned}
$$


The polynomial $P_{n}(x)$ and hence the rational function $U_{n}(x)$ satisfy a finite discrete orthogonality associated with the poles and residues of the continued fraction (4.9). From the results in Ismail and Masson [17], we know this to be

$$
\sum_{k=0}^{N} \frac{P_{n}\left(x_{k}\right) x_{k}^{m} R_{k}}{\prod_{j=2}^{n}\left(x_{k}-\alpha_{j+1}\right)\left(x_{k}-\beta_{j+1}\right)}=0, \quad 0 \leq m<n,
$$

or, equivalently,

$$
\sum_{k=0}^{N} \frac{U_{n}\left(x_{k}\right) x_{k}^{m} R_{k}}{\prod_{j=1}^{n}\left(x_{k}-\alpha_{j+1}\right)}=0, \quad 0 \leq m<n .
$$

It remains to calculate the $x_{k}$ and $R_{k}$ and then adjust the partial orthogonality $\left(4.12^{\prime}\right)$ to obtain an explicit rational biorthogonality.

The singular points $x_{k}$ are easily calculated from (4.9). They are given by the zeros of the denominator factors $\left(b e^{-\xi}, \mu b e^{\xi}\right)_{k+1}, k=0,1, \ldots, N$. This gives poles at $e^{\xi}=b q^{k}$ or $q^{-k} / \mu b, k=0,1, \ldots, N$. That is,

$$
x_{k}=\frac{\left(b q^{k}+q^{-k} / b \mu\right)}{2}, \quad k=0,1, \ldots, N .
$$

The explicit calculation of the residues $R_{k}$ is possible because they are expressed in terms of a terminating very-well-poised ${ }_{8} \phi_{7}$ for which one may use the Jackson summation formula. In detail we have

$$
\begin{aligned}
R_{k}= & C q^{-k} \lim _{e^{\xi} \rightarrow b q^{k}} \frac{\left(1-q^{k} e^{-\xi} b\right)\left(1-\mu q^{k} e^{\xi} b\right)}{\left(1-e^{-\xi} b\right)\left(1-\mu e^{\xi} b\right)} \\
& \times{ }_{10} W_{9}\left(a q ; q, \frac{a q e^{\xi}}{b}, \frac{a q e^{-\xi}}{\mu b}, \frac{a q}{d}, \frac{a q}{e}, \frac{\mu b^{2} d e q^{N}}{a}, q^{-N} ; q\right), \\
C= & \frac{u_{0} b d e(1-a q) q^{N}}{(1-d)(1-e)\left(1-a q^{N+1}\right)} .
\end{aligned}
$$

This limit gives

$$
\begin{aligned}
R_{k}=C & (-1)^{k} q^{k(k-1) / 2} \frac{\left(1-\mu q^{2 k} b^{2}\right)\left(1-a q^{2 k+1}\right)}{\left(1-\mu q^{k} b^{2}\right)(1-a q)} \\
& \times \frac{\left(a q^{k+1}, \frac{a}{\mu b^{2}} q^{-k+1}, \frac{a q}{d}, \frac{a q}{e}, q^{-N}, \frac{\mu b^{2} d e}{a} q^{N}\right)_{k}}{\left(\mu b^{2} q^{k+1}, d q, e q, a q^{N+2}, \frac{a^{2} q^{2}-N}{\mu b^{2} d e}, q\right)_{k}} \\
& \times{ }_{8} W_{7}\left(a q^{2 k+1} ; \frac{a q}{\mu b^{2}}, \frac{a q^{k+1}}{d}, \frac{a q^{k+1}}{e}, \frac{\mu b^{2} d e q^{N+k}}{a}, q^{-N+k} ; q\right) .
\end{aligned}
$$

The above ${ }_{8} W_{7}$ can be summed using the Jackson summation [7, (II. 22), p. 238] to give, after some simplification,

$$
\begin{aligned}
R_{k}= & C \frac{\left(a q^{2}, \frac{\mu b^{2} d}{a}, \frac{\mu b^{2} e}{a}, \frac{d e}{a}\right)_{N}}{\left(\mu b^{2} q, d q, e q, \frac{\mu b^{2} d e}{a^{2}}\right)_{N}} \\
& \times \frac{\left(\mu b^{2}, q \sqrt{\mu b^{2}},-q \sqrt{\mu b^{2}}, \frac{\mu b^{2}}{a}, \frac{a q}{d}, \frac{a q}{e}, \frac{\mu b^{2} d e q^{N}}{a}, q^{-N}\right)_{k}}{\left(\sqrt{\mu b^{2}},-\sqrt{\mu b^{2}}, a q, \frac{\mu b^{2} d}{a}, \frac{\mu b^{2} e}{a}, \frac{a}{d e} q^{1-N}, \mu b^{2} q^{N+1}, q\right)_{k}} .
\end{aligned}
$$


The partial orthogonality $\left(4.12^{\prime}\right)$ is now explicit. We next modify it to obtain an explicit biorthogonality.

Note that in the orthogonality expression $\left(4.12^{\prime}\right)$ there is a denominator factor

$$
x_{k}-\alpha_{2}=\frac{q^{-k}}{2 b \mu}\left(1-\frac{a}{d e} q^{1-N+k}\right)\left(1-\frac{\mu b^{2} d e}{a} q^{k+N-1}\right) .
$$

We incorporate this factor into $R_{k}$ and define a new weight

$$
\begin{aligned}
\omega_{k} & =\frac{R_{k}\left(1-\frac{a}{d e} q^{-N+1}\right)\left(1-\mu \frac{b^{2} d e}{a} q^{N-1}\right)}{R_{0}\left(x_{k}-\alpha_{2}\right) 2 \mu b} \\
& =\frac{\left(\mu b^{2}, q \sqrt{\mu b^{2}},-q \sqrt{\mu b^{2}}, \frac{\mu b^{2}}{a}, \frac{a q}{d}, \frac{a q}{e}, \frac{\mu b^{2} d e q^{N-1}}{a}\right)_{k} q^{k}}{\left(\sqrt{\mu b^{2}},-\sqrt{\mu b^{2}}, a q, \frac{\mu b^{2} d}{a}, \frac{\mu b^{2} e}{a}, \mu b^{2} q^{N+1}, \frac{a}{d e} q^{-N+2}, q\right)_{k}} .
\end{aligned}
$$

The orthogonality $\left(4.12^{\prime}\right)$ can then be restated as

$$
\sum_{k=0}^{N} \frac{U_{n}\left(x_{k}\right) Q_{m}\left(x_{k}\right) \omega_{k}}{\prod_{j=2}^{N}\left(x_{k}-\alpha_{j+1}\right)}=0, \quad 0 \leq m<n,
$$

where $Q_{m}(x)$ is any polynomial of degree $m$. We will now use a symmetry of $w_{k}$ to obtain a full biorthogonality.

Consider the parameter interchange

$$
a \leftrightarrow \frac{a q^{1-N}}{d e}
$$

with $\mu, b, \frac{a q}{d}, \frac{a q}{e}$ unchanged. It is easy to see that with (4.18) we have $\omega_{k}$ unchanged but

$$
\alpha_{j+1} \leftrightarrow \beta_{j}
$$

With (4.18) we also have

$$
U_{n}(x) \leftrightarrow V_{n}(x),
$$

where

$$
V_{n}(x)={ }_{10} W_{9}\left(\frac{a q^{1-N}}{d e} ; b e^{-\xi}, \mu b e^{\xi}, \frac{q^{1-N}}{d}, \frac{q^{1-N}}{e}, \frac{a q^{2}}{d e}, \frac{a^{2} q^{1-N+n}}{\mu b^{2} d e}, q^{-n} ; q\right) .
$$

It follows from (4.17)-(4.21) that we have a full biorthogonality

$$
\sum_{k=0}^{N} U_{n}\left(x_{k}\right) V_{m}\left(x_{k}\right) \omega_{k}=0, \quad n \neq m .
$$

It remains to calculate the $n=m$ case to obtain

$$
W_{n}:=\sum_{k=0}^{n} U_{n}\left(x_{k}\right) V_{n}\left(x_{k}\right) \omega_{k}
$$

This, the most tedious part of the calculation, we now outline. 
From Ismail and Masson [17] we have a fundamental expression for the Stieltjes transform:

$$
\begin{aligned}
& \sum_{k=0}^{N} \frac{Q_{m}\left(x_{k}\right) P_{n}\left(x_{k}\right) R_{k}}{\left[\prod_{j=1}^{n}\left(x_{k}-\alpha_{j+1}\right)\left(x_{k}-\beta_{j+1}\right)\right]\left(x-x_{k}\right)} \\
& \quad=\frac{Q_{m}(x) Y_{n}^{(\min )}(x)}{\lambda_{1} Y_{-1}^{(\min )}(x)\left[\prod_{j=0}^{n}\left(x-\alpha_{j+1}\right)\left(x-\beta_{j+1}\right)\right]}, \quad 0 \leq m<n,
\end{aligned}
$$

where $Y_{n}^{(\min )}$ is the minimal solution to the recurrence $\left(4.3^{\prime}\right)$ and $Q_{m}(x)$ is any polynomial of degree $m<n$. The calculation of $W_{n}$ in (4.23) will require an evaluation of the right side of (4.24) at $x=\alpha_{n+2}$ for $m=n$.

We first note that

$$
\frac{P_{n}\left(x_{k}\right)}{\prod_{j=1}^{n}\left(x_{k}-\beta_{j+1}\right)}=\frac{D_{n} U_{n}\left(x_{k}\right)}{\prod_{k=-1}^{n} u_{k}}
$$

where

$$
D_{n}=\left(-\frac{2 a}{b}\right)^{n} q^{n(n+1)} \frac{\left(\frac{s}{q}, \frac{s}{a q}, \frac{a q}{d}, \frac{a q}{e}, q^{-N}\right)_{n}}{\left(\frac{s}{q}\right)_{2 n}(a q)_{n}} .
$$

Secondly, we may write

$$
V_{n}\left(x_{k}\right)=\frac{E_{n} Q_{n}\left(x_{k}\right)}{\prod_{j=1}^{n}\left(x_{k}-\alpha_{j+2}\right)}+\text { additional terms }
$$

where

$$
\begin{aligned}
E_{n} & =q^{-n^{2}}\left(\frac{a q}{2 \mu b s}\right)^{n} \frac{\left(1-\frac{a q^{2 n-N+1}}{d e}\right)}{\left(1-\frac{a q^{-N+1}}{d e}\right)} \frac{\left(\frac{q^{-N+1}}{d}, \frac{q^{-N+1}}{e}, \frac{a q^{2}}{d e}, s q^{n-1}, \frac{a q^{-N+1}}{d e}\right)_{n}}{\left(\frac{a q}{d}, \frac{a q}{e}, q^{-N}, \frac{a q^{3-N-n}}{d e s}, \frac{a q^{2+n-N}}{d e}\right)_{n}} \\
Q_{n}(x) & =\left(b e^{-\xi}, \mu b e^{\xi}\right)_{n} .
\end{aligned}
$$

Note that the additional terms in (4.27) do not contribute to the right side of (4.24) evaluated at $x=\alpha_{n+2}$, because they have a numerator factor $\left(x-\alpha_{n+2}\right)$. From (4.24)-(4.28) it now follows that (4.23) can be calculated, since

$$
W_{n}=-\frac{E_{n} F_{n} G_{n}\left(1-a q^{-N+1}\right)\left(1-\mu b^{2} \frac{d e}{a} q^{N-1}\right) u_{0}}{2 D_{n} H_{n} R_{0} \mu b},
$$

where $E_{n}$ and $D_{n}$ are given by (4.26) and (4.28),

$$
\begin{gathered}
R_{0}=-\frac{u_{0} a q(1-a q)\left(a q^{2}, \frac{\mu b^{2} d}{a}, \frac{\mu b^{2} e}{a}, \frac{d e}{a}\right)_{N}}{2 \mu b s(1-d)(1-e)\left(1-a q^{N+1}\right)\left(\mu b^{2} q, d q, e q, \frac{\mu b^{2} d e}{a^{2} q}\right)_{N}}, \\
G_{n}=\left.\left(b e^{-\xi}, \mu b e^{\xi}\right)_{n}\right|_{x=\alpha_{n+2}}=\left(\frac{a}{s} q^{1-n}, \frac{\mu b^{2} s}{a} q^{n-1}\right)_{n},
\end{gathered}
$$




$$
\begin{aligned}
H_{n} & =\left.\prod_{k=1}^{n}\left(x-\alpha_{k+1}\right)\left(x-\beta_{k+1}\right)\right|_{x=\alpha_{n+2}} \\
& =\left(\frac{q^{2}}{4 \mu s}\right)^{n} q^{-n(n+1)}\left(q^{-n}, \frac{\mu b^{2} s^{2}}{a^{2}} q^{n}, s q^{n}, \frac{a^{2} q^{-n+2}}{\mu b^{2} s}\right)_{n},
\end{aligned}
$$

and

$$
F_{n}=\left.\frac{X_{n}^{(\min )}(x)}{\gamma_{0} X_{-1}^{(\min )}(x)\left(x-\alpha_{1}\right)\left(x-\beta_{1}\right)}\right|_{x=\alpha_{n+2}} .
$$

From the minimal solution expression (3.30), with the replacement (4.1), we find that

$$
\begin{aligned}
F_{n}= & \frac{q^{-1}\left(1-s q^{2 n-1}\right)(1-a q)\left(\frac{q^{2} a}{s}\right)^{n+1}}{\left(1-\frac{s}{q}\right)\left(1-\frac{a}{s} q^{1-n}\right)\left(1-\mu \frac{b^{2}}{a} s q^{n-1}\right)(1-d)(1-e)\left(1-a q^{N+1}\right)} \\
& \times \frac{\left(q, q^{-n}, \frac{\mu b^{2} s^{2}}{a^{2}} q^{n-2}, \frac{d s}{a q}, \frac{e s}{a q}, s q^{N}, s q^{n}, \frac{a^{2} q^{2-n}}{\mu b^{2} s}, \frac{a q}{d}, \frac{a q}{e}, q^{-N}, \frac{s}{q}\right)_{n}\left(a q^{2}\right)_{2 n}}{(s, s)_{2 n}\left(\frac{a q^{2-n}}{s}, \frac{\mu b^{2} s q^{n}}{a}, d q, e q, a q^{N+2}, a q\right)_{n}} \\
& \times{ }_{8} W_{7}\left(a q^{2 n+1} ; q^{n+1}, \frac{a^{2} q^{2}}{\mu b^{2} s}, \frac{a q^{n+1}}{d}, \frac{a q^{n+1}}{e}, q^{-N+n} ; q\right) .
\end{aligned}
$$

Using the Jackson summation for this last ${ }_{8} W_{7}$ and putting all factors together, we finally obtain, after much simplification,

$$
\begin{aligned}
W_{n}= & q^{-n} \frac{\left(q, \frac{a q^{1-N}}{\mu b^{2} d}, \frac{a q^{1-N}}{\mu b^{2} e}, \frac{a^{2} q^{2}}{\mu b^{2} e}, \frac{a q^{2-N}}{d e}, a q\right)_{n}}{\left(q^{N}, \frac{a q^{1-N}}{\mu b^{2} d e}, \frac{a q}{d}, \frac{a q}{e}, \frac{a}{\mu b^{2}}, \frac{a^{2} q^{2-N}}{\mu b^{2} d e}\right)_{n}} \\
& \times \frac{\left(\mu b^{2} q, d, e, \frac{\mu b^{2} d e}{a^{2} q}\right)_{N}\left(1-\frac{a^{2} q^{1-N+n}}{\mu b^{2} d e}\right)}{\left(a q, \frac{\mu b^{2} d}{a}, \frac{\mu b^{2} e}{a}, \frac{d e}{a q}\right)_{N}\left(1-\frac{a^{2} q^{1-N+2 n}}{\mu b^{2} d e}\right)} .
\end{aligned}
$$

We now summarize all of the above but with a normalized probability measure. That is, we choose

$$
r_{k}:=\frac{\omega_{k}}{W_{0}}
$$

so that

$$
\sum_{k=0}^{N} r_{k}=1
$$

Theorem 4.1. Let $x=\left(e^{\xi}+\mu^{-1} e^{-\xi}\right) / 2$,

$$
\begin{aligned}
& U_{n}(x)={ }_{10} W_{9}\left(a ; b e^{-\xi}, \mu b e^{\xi}, d, e, a q^{N+1}, \frac{a^{2} q^{1-N+n}}{\mu b^{2} d e}, q^{-n} ; q\right), \\
& V_{m}(x)={ }_{10} W_{9}\left(\frac{a q^{1-N}}{d e} ; b e^{-\xi}, \mu b e^{\xi}, \frac{q^{1-N}}{d}, \frac{q^{1-N}}{e}, \frac{a q^{2}}{d e}, \frac{a^{2} q^{1-N+m}}{\mu b^{2} d e}, q^{-m} ; q\right) .
\end{aligned}
$$


Then

$$
\sum_{k=0}^{N} U_{n}\left(x_{k}\right) V_{m}\left(x_{k}\right) r_{k}=C_{n} \delta_{n, m}, \quad 0 \leq n, m \leq N
$$

where

$$
\begin{aligned}
x_{k}= & \left(b q^{k}+q^{-k} / b \mu\right) / 2 \\
r_{k}= & q^{k} \frac{\left(\mu b^{2}, q \sqrt{\mu b^{2}},-q \sqrt{\mu b^{2}}, \frac{\mu b^{2}}{a}, \frac{a q}{d}, \frac{a q}{e}, \frac{\mu b^{2} d e}{a} q^{N-1}, q^{-N}\right)_{k}}{\left(\sqrt{\mu b^{2}},-\sqrt{\mu b^{2}}, a q, \frac{\mu b^{2} d}{a}, \frac{\mu b^{2} e}{a}, \mu b^{2} q^{N+1}, \frac{a q^{2-N}}{d e}, q\right)_{k}} \\
& \times \frac{\left(a q, \frac{\mu b^{2} d}{a}, \frac{\mu b^{2} e}{a}, \frac{d e}{a q}\right)_{N}}{\left(\mu b^{2} q, d, e, \frac{\mu b^{2} d e}{a^{2} q}\right)_{N}} \\
C_{n}= & q^{-n} \frac{\left.\left(q, \frac{a q^{1-N}}{\mu b^{2} d}, \frac{a q^{1-N}}{\mu b^{2} e}, \frac{a^{2} q^{2}}{\mu b^{2} d e}, \frac{a q^{2-N}}{d e}, a q^{N}\right)_{n} \frac{\left(1-\frac{a^{2} q^{1-N+n}}{\mu b^{2} d e}\right)}{\left(q^{-N}, \frac{a q^{1-N}}{\mu b^{2} d e}, \frac{a q}{d}, \frac{a q}{e}, \frac{a}{\mu b^{2}}, \frac{a^{2} q^{2}-N}{\mu b^{2} d e}\right)_{n}} . \frac{a^{2} q^{1-N+2 n}}{\mu b^{2} d e}\right)}{(1-}
\end{aligned}
$$

We have previously stated Theorem 4.1 , without proof, for the special case $\mu=$ -1 [25]. Wilson, using a different method, derived Theorem 4.1 (and its $q \rightarrow 1$ limit) for the special case $\mu=1$ [31] but with a misprint. (In $C_{n}$ the factor $q^{-n}$ was omitted and the numerator factor $(a q)_{N}$ in $r_{k}$ was incorrectly written as $\left(a^{2} q\right)_{N}$.) Rahman and Suslov [26], with a still different approach, obtained a general biorthogonality which we have checked to be equivalent to Theorem 4.1 and its $q \rightarrow 1$ limit given in Corollary 4.7 below. In order to do the calculations needed to compare Theorem 4.1 with the results in [26] we used some additional notes that were given to us by S.K. Suslov [28]. These notes also contained some limit cases that were derived independently by Suslov.

The two main ingredients in our derivation of Theorem 4.1 were the continued fraction (3.34), which came from a three-term recurrence for ${ }_{10} \phi_{9}$ 's and the Jackson ${ }_{8} \phi_{7}$ summation formula, which is also the " $q$-beta integral" in (4.37) (the $n=m=0$ case). This gives a $q$-version of Askey's conjecture [1, p. 37] of a connection between Ramanujan's Entry 40, Dougall's ${ }_{7} F_{6}$ summation formula and a ${ }_{9} F_{8}$ three-term recurrence. See also the discussion after Corollary 3.3.

We now state six limit cases of Theorem 4.1 as corollaries. The first five are at the ${ }_{4} \phi_{3}$ (or very-well- poised ${ }_{8} \phi_{7}$ ) level, while the final one is a $q \rightarrow 1$ limit at the ${ }_{9} F_{8}$ level. Further lower level limits may be taken. This will result in an Askey type scheme for discrete rational biorthogonality analogous to that for hypergeometric orthogonal polynomials [20]. This new scheme of orthogonality makes contact with the polynomial scheme through Corollary 4.6, which is the case of $q$-Racah polynomials.

$4 \mathrm{~b})$. The ${ }_{4} \phi_{3}$ level. We give five limiting cases of Theorem 4.1. The calculations are straightforward and will not be detailed. 


\section{Corollary 4.2. Let}

$$
\begin{aligned}
U_{n}(x) & =\frac{\left(a q, \frac{a q}{d e}\right)_{n}}{\left(\frac{a q}{d}, \frac{a q}{e}\right)_{n}}{ }_{4} \phi_{3}\left(\begin{array}{c}
\frac{2 x q^{-N}}{b}, d, e, q^{-n} \\
\frac{2 a q x}{b}, q^{-N}, \frac{d e q^{-n}}{a} ; q
\end{array}\right), \\
V_{m}(x) & =\frac{\left(\frac{a q^{-N+2}}{d e}, a q^{N}\right)_{m}}{\left(\frac{a q}{e}, \frac{a q}{d}\right)_{m}} \phi_{3}\left(\frac{2 x q^{-N}}{b}, \frac{q^{-N+1}}{\frac{2 a x q^{-N+2}}{b d e}}, \frac{q^{-N+1}}{e}, q^{-N}, \frac{q^{-N}-m+1}{a} ; q\right) .
\end{aligned}
$$

Then

$$
\sum_{k=0}^{N} U_{n}\left(x_{k}\right) V_{m}\left(x_{k}\right) r_{k}=C_{n} \delta_{n, m}
$$

where $x_{k}=\frac{1}{2} b q^{k}$,

$$
r_{k}=\frac{\left(\frac{a q}{d}, \frac{a q}{e}, q^{-N}\right)_{k}}{\left(a q, \frac{a q^{2-N}}{d e}, q\right)_{k}} q^{k} \frac{\left(a q, \frac{d e}{a q}\right)_{N}}{(d, e)_{N}}
$$

and

$$
C_{n}=q^{-n} \frac{\left(q, a q, \frac{a q^{2-N}}{d e}\right)_{n}}{\left(q^{-N}, \frac{a q}{d}, \frac{a q}{e}\right)_{n}} .
$$

Proof. Take the limit as $\mu \rightarrow \infty$ in Theorem 4.1. The limiting $U_{n}$ and $V_{m}$ are terminating ${ }_{8} \phi_{7}$ 's. We then use Watson's transformation formula [7, (III. 18), p. 242] to obtain the above ${ }_{4} \phi_{3}$ expressions for $U_{n}(x)$ and $V_{m}(x)$.

Corollary 4.3. Let $x=\frac{1}{2}\left(e^{\xi}+\mu^{-1} e^{-\xi}\right)$,

$$
\begin{aligned}
U_{n}(x) & =\frac{\left(a q, \frac{a q}{\mu b^{2}}\right)_{n}}{\left(\frac{a q}{\mu b} e^{-\xi}, \frac{a q}{b} e^{\xi}\right)_{n}}{ }_{4} \phi_{3}\left(\begin{array}{c}
\frac{a q}{d e}, b e^{-\xi}, \mu b e^{\xi}, q^{-n} \\
\frac{a q}{d}, \frac{a q}{e}, \frac{\mu b^{2}}{a} q^{-n} ; q
\end{array}\right), \\
V_{m}(x) & ={ }_{4} \phi_{3}\left(\begin{array}{c}
b e^{-\xi}, \mu b e^{\xi}, \frac{a q^{2}}{d e}, q^{-m} \\
\frac{a q}{e}, \frac{a q}{d}, \frac{\mu b^{2}}{a} q^{1-m}
\end{array} ; q\right) .
\end{aligned}
$$

Then

$$
\sum_{k=0}^{\infty} U_{n}\left(x_{k}\right) V_{m}\left(x_{k}\right) r_{k}=C_{n} \delta_{n, m},
$$

where

$$
\begin{aligned}
& x_{k}=\frac{1}{2}\left(b q^{k}+q^{-k} / b \mu\right) \\
& r_{k}=\frac{\left(\mu b^{2}, q \sqrt{\mu b^{2}},-q \sqrt{\mu b^{2}}, \frac{\mu b^{2}}{a}, \frac{a q}{d}, \frac{a q}{e}\right)_{k}}{\left(\sqrt{\mu b^{2}},-\sqrt{\mu b^{2}}, a q, \frac{\mu b^{2} d}{a}, \frac{\mu b^{2} e}{a}, q\right)_{k}}\left(\frac{d e}{a q}\right)^{k} \frac{\left(a q, \frac{\mu b^{2} d}{a}, \frac{\mu b^{2} e}{a}, \frac{d e}{a q}\right)_{\infty}}{\left(\mu b^{2} q, d, e, \frac{\mu b^{2} d e}{a^{2} q}\right)_{\infty}}
\end{aligned}
$$

and

$$
C_{n}=\frac{\left(q, \frac{a^{2} q^{2}}{\mu b^{2} d e}, a q\right)_{n}}{\left(\frac{a q}{d}, \frac{a q}{e}, \frac{a}{\mu b^{2}}\right)_{n} .}
$$


Proof. Take the limit as $N \rightarrow \infty$ in Theorem 4.1 and use Watson's transformation formula.

Corollary 4.4. Let $x=\frac{1}{2}\left(e^{\xi}+\mu^{-1} e^{-\xi}\right)$,

$$
\begin{aligned}
& U_{n}(x)=\frac{1}{d^{n}} \frac{\left(a q, \frac{a q}{\mu b^{2} e}\right)_{n}}{\left(\frac{a q}{d}, \frac{a q}{\mu b^{2} d e}\right)_{n}} \phi_{3}\left(\begin{array}{l}
\frac{a q}{\mu b^{2}}, d, \frac{a^{2} q^{1+n}}{\mu b^{2} d e}, q^{-n} \\
\frac{a q}{b} e^{\xi}, \frac{a q}{b \mu} e^{-\xi}, \frac{a q}{\mu b^{2} e}
\end{array} ; q\right), \\
& V_{m}(x)=\left(\frac{e}{q}\right)^{m} \frac{\left(\frac{a q^{2}}{d e}, \frac{a q}{\mu b^{2} e}\right)_{m}}{\left(\frac{a q}{d}, \frac{a}{\mu b^{2}}\right)_{m}} \phi_{3}\left(\begin{array}{c}
\frac{a q^{2}}{\mu b^{2} d e}, \frac{q}{e}, \frac{a^{2} q^{m+1}}{\mu b^{2} d e}, q^{-m} \\
\frac{a q^{2}}{b d e} e^{\xi}, \frac{a q^{2}}{\mu b d e} e^{-\xi}, \frac{a q}{\mu b^{2} e}
\end{array} ; q\right) .
\end{aligned}
$$

Then

$$
\sum_{k=0}^{\infty} U_{n}\left(x_{k}\right) V_{m}\left(x_{k}\right) r_{k}=C_{n} \delta_{m, n}
$$

where

$$
\begin{aligned}
& x_{k}=\frac{1}{2}\left(b q^{k}+\frac{q^{-k}}{b \mu}\right), \\
& r_{k}=\frac{\left(\mu b^{2}, q \sqrt{\mu b^{2}},-q \sqrt{\mu b^{2}}, \frac{\mu b^{2}}{a}, \frac{a q}{d}, \frac{\mu b^{2} d e}{a q}\right)_{k}}{\left(\sqrt{\mu b^{2}},-\sqrt{\mu b^{2}}, a q, \frac{\mu b^{2} d}{a}, \frac{a q^{2}}{d e}, q\right)_{k}}\left(\frac{a q}{\mu b^{2} e}\right)^{k} \frac{\left(a q, \frac{\mu b^{2} d}{a}, \frac{a q}{\mu b^{2} e}, \frac{a q}{d}\right)_{\infty}}{\left(\mu b^{2} q, d, \frac{q}{e}, \frac{a^{2} q^{2}}{\mu b^{2} d e}\right)_{\infty}}
\end{aligned}
$$

and

$$
C_{n}=\frac{\left(q, \frac{a q}{\mu b^{2} e}, \frac{a q^{2}}{d e}, a q\right)_{n}}{\left(\frac{a q}{\mu b^{2} d e}, \frac{a q}{d}, \frac{a}{\mu b^{2}}, \frac{a^{2} q^{2}}{\mu b^{2} d e}\right)_{n}}\left(\frac{a}{\mu b^{2} d}\right)^{n} \frac{\left(1-\frac{a^{2} q^{1+n}}{\mu b^{2} d e}\right)}{\left(1-\frac{a^{2} q^{1+2 n}}{\mu b^{2} d e}\right)}
$$

Proof. Replace $e$ by $e q^{-N}$ in Theorem 4.1, take the limit as $N \rightarrow \infty$ and use Watson's transformation formula.

Corollary 4.5. Let

$$
\begin{aligned}
& U_{n}(x)=\frac{\left(a q, \frac{2 a q x}{b d}\right)_{n}}{\left(\frac{a q}{d}, \frac{2 a q x}{b}\right)_{n}} \phi_{3}\left(\begin{array}{c}
\frac{b^{2} \mu d e}{a^{2} q} q^{-n}, d, \frac{b}{2 x}, q^{-n} \\
\frac{b^{2} \mu d e}{a} q^{N-n}, q^{-N}, \frac{b d}{2 a x} q^{-n}
\end{array} ; q\right),
\end{aligned}
$$

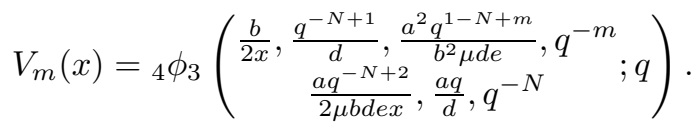

Then

$$
\sum_{k=0}^{N} U_{n}\left(x_{k}\right) V_{m}\left(x_{k}\right) r_{k}=C_{n} \delta_{n, m}
$$

where $x_{k}=\frac{1}{2} b q^{k}$,

$$
r_{k}=\frac{\left(\frac{a q}{d}, q^{-N}, \frac{\mu b^{2} d e}{a q} q^{N}\right)_{k}}{\left(a q, \frac{\mu b^{2} e}{a}, q\right)_{k}} q^{k}\left(\frac{d}{a q}\right)^{N} \frac{\left(a q, \frac{\mu b^{2} e}{a}\right)_{N}}{\left(d, \frac{\mu b^{2} d e}{a^{2} q}\right)_{N}},
$$


and

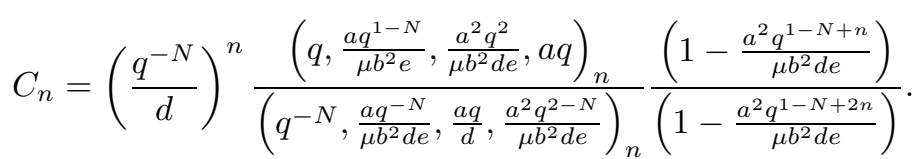

Proof. Replace $e$ and $\mu$ by $e q^{M}$ and $\mu q^{-M}$ respectively in Theorem 4.1, take the limit as $M \rightarrow \infty$ and use Watson's transformation formula.

Corollary 4.6. Let $x=\frac{1}{2}\left(e^{\xi}+\mu^{-1} e^{-\xi}\right)$,

$$
\begin{gathered}
U_{n}(x)={ }_{4} \phi_{3}\left(\begin{array}{c}
\left.b e^{-\xi}, \mu b e^{\xi}, \frac{a^{2} q^{1-N+n}}{\mu b^{2} d e}, q^{-n} ; q\right), \\
\frac{a q}{d}, \frac{a q}{e}, q^{-N}
\end{array}\right. \\
V_{m}(x)={ }_{4} \phi_{3}\left(\begin{array}{c}
\left.b e^{-\xi}, \mu b e^{\xi}, \frac{a^{2} q^{1-N+m}}{\mu b^{2} d e}, q^{-m} ; q\right) . \\
\frac{a q}{d}, \frac{a q}{e}, q^{-N}
\end{array}\right.
\end{gathered}
$$

Then

$$
\sum_{k=0}^{N} U_{n}\left(x_{k}\right) V_{m}\left(x_{k}\right) r_{k}=C_{n} \delta_{n, m}
$$

where $x_{k}=\frac{1}{2}\left(b q^{k}+\frac{q^{-k}}{b \mu}\right)$,

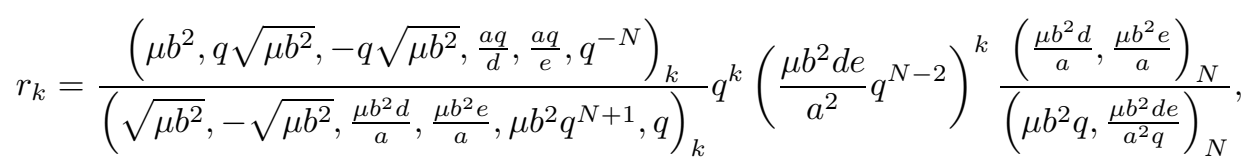

and

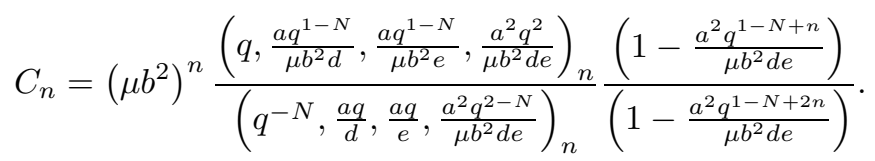

Proof. Replace $a, d, e$ by $a q^{-M}, d q^{-M}, e q^{-M}$ respectively in Theorem 4.1 and take the limit as $M \rightarrow \infty$.

Note that Corollary 4.6 corresponds to the case of $q$-Racah polynomials, where $U_{n}(x)=V_{n}(x)$ are polynomials in $x$ of degree $n$.

$4 \mathrm{c})$. The top ${ }_{9} F_{8}$ level. The $q \rightarrow 1$ limit of Theorem 4.1 yields

Corollary 4.7. Let $x=u(u+\mu)$,

$$
\begin{gathered}
U_{n}(x)={ }_{9} F_{8}\left[\begin{array}{c}
a, 1+\frac{1}{2} a, b-u, b+u+\mu, d, e, a+N+1, \\
\frac{1}{2} a, a-b+1+u, a-b-\mu+1-u, a-d+1, a-e+1,-N, \\
2 a+1-N-2 b-d-e-\mu+n,-n \\
2 b+\nu+d+e-a+N-n, a+n+1
\end{array} ;\right. \\
V_{m}(x)={ }_{9} F_{8}\left[\begin{array}{c}
a-d-e-N+1,1+\frac{1}{2}(a-d-e-N+1), b-u, b+\mu+u, \\
\frac{1}{2}(a-d-e-N+1), a-b-d-e-N+2+u, \\
-N-d+1,-N-e+1, a-d-e+2, \\
a-\mu-b-d-e-N+2-u, a-e+1, a-d+1,-N, \\
2 a+1-N-2 b-d-e-\mu+m,-m \\
2 b-a+\mu-m+1, a-d-e-N+2+m
\end{array} ; .\right.
\end{gathered}
$$


Then

$$
\sum_{k=0}^{N} U_{n}\left(x_{k}\right) V_{m}\left(x_{k}\right) r_{k}=C_{n} \delta_{n, m}
$$

where

$$
\begin{aligned}
x_{k}= & (b+k)(b+k+\mu), \\
& \left(\mu+2 b, 1+\frac{1}{2}(\mu+2 b), \mu+2 b-a, a-d+1,\right. \\
r_{k}= & \frac{a-e+1,-N, \mu+2 b+d+e-a+N-1)_{k}}{\left(\frac{1}{2}(\mu+2 b), a+1, \mu+2 b+d-a, \mu+2 b+e-a,\right.} \\
& \times \frac{(a+2 b+N+1, a-d-e+2-N, 1)_{k}}{(\mu+2 b+d-a, \mu+2 b+e-a, d+e-a-1)_{N}}, \\
& \quad
\end{aligned}
$$

and

$$
\begin{aligned}
C_{n}= & \frac{(1, a+1-N-\mu-2 b-d, a+1-N-\mu-2 b-e)_{n}}{(-N, a+1-N-\mu-2 b-d-e, a-d+1)_{n}} \\
& \times \frac{(2 a+2-\mu-2 b-d-e, a+2-N-d-e, a+1)_{n}}{(a-e+1, a-\mu-2 b, 2 a+2-N-\mu-2 b-d-e)_{n}} .
\end{aligned}
$$

Proof. In the orthogonality of Theorem 4.1, we replace $a, b, d, e, \mu$ by $q^{a}, q^{b}, q^{d}$, $q^{e}, q^{\mu}$ and take the limit as $q \rightarrow 1$. Note that in the statement of Corollary 4.7, $(a, b, \ldots, c)_{k}$ is now the usual multiple shifted factorial and ${ }_{9} F_{8}$ is a generalized hypergeometric function (see Bailey [4]).

For a different derivation of the $\mu=0$ case of Corollary 4.7, see Wilson [31].

\section{5. $\mathrm{AN}_{8} \phi_{7}$ MODEL}

If we replace $f$ by $F q^{M}$ and $s$ by $\frac{a^{3} q^{3} q^{-M}}{b c d e F}$ in equation (3.2) and let $M \rightarrow \infty$, then (3.2) reduces to the equation

$$
\begin{aligned}
& Y_{n+1}-c_{n} Y_{n}+d_{n} Y_{n-1}=0 \\
& c_{n}=C_{n}+D_{n}+\frac{a^{2} q^{2 n+2}}{b c d e h^{2}} \frac{(1-b)(1-c)(1-d)(1-e)}{\left(1-\frac{a q^{n+1}}{h}\right)} \\
& d_{n}=C_{n-1} D_{n} \\
& C_{n}=-\frac{\left(1-\frac{a q^{n+1}}{b h}\right)\left(1-\frac{a q^{n+1}}{c h}\right)\left(1-\frac{a q^{n+1}}{d h}\right)\left(1-\frac{a q^{n+1}}{e h}\right)}{\left(1-\frac{a q^{n+1}}{h}\right)}, \\
& D_{n}=-q\left(1-\frac{q^{n}}{h}\right)\left(1-\frac{a q^{n}}{h}\right)\left(1-\frac{a^{2} q^{n+1}}{b c d e h}\right) .
\end{aligned}
$$

In [12], (5.1) was derived from a three-term contiguous relation for a very-wellpoised ${ }_{8} \phi_{7}$ series in the particular case $h=1$. Following the calculation in [12], we 
have the following four solutions of (5.1):

$$
\begin{aligned}
Y_{n}^{(1)}= & \frac{(-1)^{n}\left(\frac{a q^{n+1}}{h}\right)_{\infty}}{\left(\frac{a q^{n+1}}{b h}, \frac{a q^{n+1}}{c h}, \frac{a q^{n+1}}{d h}, \frac{a q^{n+1}}{e h}\right)_{\infty}}{ }_{8} W_{7}\left(a ; b, c, d, e, h q^{-n} ; \frac{a^{2} q^{n+2}}{b c d e h}\right), \\
Y_{n}^{(2)}= & \frac{(-1)^{n}}{\left(\frac{q^{n+1}}{h}, \frac{a^{2} q^{n+2}}{b c d e h}, \frac{a q^{n}}{h}\right)_{\infty} W_{7}\left(\frac{q}{a} ; \frac{q}{b}, \frac{q}{c}, \frac{q}{d}, \frac{q}{e}, \frac{q^{n+1}}{h} ; \frac{b c d e h}{a^{2} q} q^{-n}\right),} \\
Y_{n}^{(3)}= & \frac{(-1)^{n}\left(\frac{a q^{n+1}}{h}\right)_{\infty}}{\left(\frac{a q^{n+1}}{b h}, \frac{a q^{n+1}}{c h}, \frac{a q^{n+1}}{d h}, \frac{a q^{n+1}}{e h}\right)_{\infty}} \\
& \times{ }_{8} W_{7}\left(\frac{b c d e}{a q} ; b, c, d, e, \frac{b c d e h q^{-n-1}}{a^{2}} ; \frac{q^{n+1}}{h}\right),
\end{aligned}
$$

and

$$
Y_{n}^{(4)}=\frac{(-1)^{n}}{\left(\frac{q^{n+1}}{h}, \frac{a^{2} q^{n+2}}{b c d e h}, \frac{a q^{n}}{h}\right)_{\infty}}{ }_{\infty} W_{7}\left(\frac{a q^{2}}{b c d e} ; \frac{q}{b}, \frac{q}{c}, \frac{q}{d}, \frac{q}{e}, \frac{a^{2} q^{n+2}}{b c d e h} ; h q^{-n}\right) .
$$

In addition to the above four solutions, two more solutions may be obtained as follows. In (5.1) we make the parameter replacements

$$
(a, b, c, d, e, h) \rightarrow\left(\frac{B^{2}}{A}, B, \frac{B C}{A}, \frac{B D}{A}, \frac{B E}{A}, \frac{B H}{A}\right)
$$

and renormalize so as to arrive at (5.1) with lower case letters $a, b, c, d, e, h$ replaced by capitals. Consequently we obtain a fifth solution to (5.1), viz.,

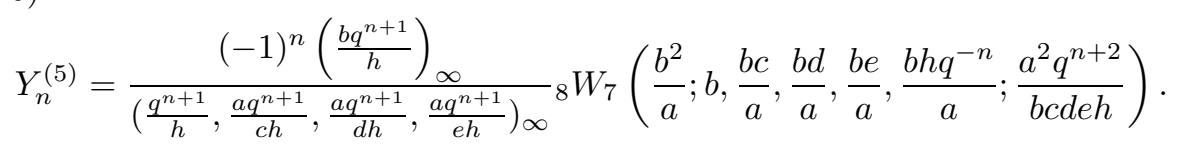

The 'reflection transformation' (see [12]) applied to (5.1) and (5.6) yields another solution:

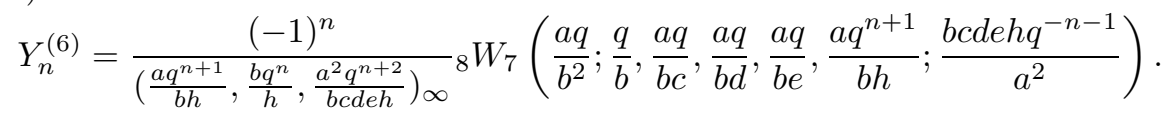

Parameter interchanges $b \leftrightarrow c, d$ or $e$ in (5.6) and (5.7) give six more solutions. Thus we have twelve pairwise linearly independent solutions to the three-term recurrence (5.1).

All these solutions may be derived as limiting cases of our $\Phi$ solutions obtained in Section 3. Thus it is easily seen that $Y_{n}^{(1)}, Y_{n}^{(2)}, Y_{n}^{(3)}, Y_{n}^{(4)}, Y_{n}^{(5)}$ and $Y_{n}^{(6)}$ are limiting cases of the solutions $X_{n}^{(1), \frac{s}{h} q^{n-1}}, X_{n}^{(2), \frac{h}{s} q^{-n+2}}, X_{n}^{(4), \frac{a q^{2}}{s}}, X_{n}^{(3), \frac{s}{a q}}$, $X_{n}^{(3), \frac{b s}{a h} q^{n-1}}$ and $X_{n}^{(4), \frac{a h}{b s} q^{-n+2}}$ respectively. Limits of all the remaining $X_{n}$ solutions are either one of the $Y_{n}$ solutions or a linear combination of the $Y_{n}$ solutions; e.g., it can be shown that $X_{n}^{(1), b}$ gives a linear combination of $\left(Y_{n}^{(1)}, Y_{n}^{(5)}\right)$; $X_{n}^{(1), h q^{-n}}$ of $\left(Y_{n}^{(1)}, Y_{n}^{(4)}\right) ; X_{n}^{(2), \frac{q}{b}}$ of $\left(Y_{n}^{(2)}, Y_{n}^{(6)}\right) ; X_{n}^{(2), \frac{q^{n+1}}{h}}$ of $\left(Y_{n}^{(2)}, Y_{n}^{(3)}\right) ; X_{n}^{(5), \frac{a q n+1}{b h}}$ of $\left(Y_{n}^{(3)}, Y_{n}^{(6)}\right) ; X_{n}^{(6), \frac{b h}{a} q^{-n}}$ of $\left(Y_{n}^{(4)}, Y_{n}^{(5)}\right) ; X_{n}^{(7), \frac{b c}{a}}$ of $\left(Y_{n}^{(5)}\right.$, a $b \leftrightarrow c$ interchange of $\left.Y_{n}^{(5)}\right) ; X_{n}^{(8), \frac{a q}{b c}}$ of $\left(Y_{n}^{(6)}\right.$, a $b \leftrightarrow c$ interchange of $\left.Y_{n}^{(6)}\right)$, etc. 
Any three of the twelve solutions of (5.1) are connected by a three-term ${ }_{8} \phi_{7}$ transformation formula. It can be shown that the connection is provided either by the standard three-term ${ }_{8} \phi_{7}$ formula ([7], (III. 37), p. 246) or by its iterate which we state below explicitly in a form suited to our purpose:

$$
\begin{aligned}
{ }_{8} W_{7}\left(a ; b, c, d, e, f ; \frac{a^{2} q^{2}}{b c d e f}\right) \\
=\frac{\left(a q, b, \frac{b}{a}, \frac{b q}{a}, \frac{c q}{a}, \frac{d q}{a}, \frac{e q}{a}, \frac{f q}{a}, \frac{a q}{c d}, \frac{a q}{c e}, \frac{a q}{c f}, \frac{a q}{d e}, \frac{a q}{d f}, \frac{a q}{e f}, \frac{b c d e f}{a^{2} q}\right)_{\infty}}{\left(\frac{q^{2}}{a}, \frac{q}{c}, \frac{q}{d}, \frac{q}{e}, \frac{q}{f}, \frac{a q}{c}, \frac{a q}{d}, \frac{a q}{e}, \frac{a q}{f}, \frac{b c}{a}, \frac{b d}{a}, \frac{b e}{a}, \frac{b f}{a}, \frac{c d e f}{a^{2}}, \frac{a^{2} q}{c d e f}\right)_{\infty}} \\
\quad \times{ }_{8} W_{7}\left(\frac{q}{a} ; \frac{q}{b}, \frac{q}{c}, \frac{q}{d}, \frac{q}{e}, \frac{q}{f} ; \frac{b c d e f}{a^{2} q}\right) \\
+\quad \frac{\left(a q, c, \frac{c}{a}, \frac{b q}{a}, \frac{b q}{d}, \frac{b q}{e}, \frac{b q}{f}, \frac{a q}{b d}, \frac{a q}{b e}, \frac{a q}{b f}\right)_{\infty}}{\left(\frac{b^{2} q}{a}, \frac{q}{d}, \frac{q}{e}, \frac{q}{f}, \frac{c}{b}, \frac{a q}{b}, \frac{a q}{d}, \frac{a q}{e}, \frac{a q}{f}, \frac{b c}{a}\right)_{\infty}} \\
\times\left[1-\frac{\left(b, \frac{q}{b}, \frac{a}{b}, \frac{b q}{a}, \frac{c d}{a}, \frac{a q}{c d}, \frac{c e}{a}, \frac{a q}{c e}, \frac{c f q}{a}, \frac{a}{c f}, \frac{b d e f}{a^{2}}, \frac{a^{2} q}{b d e f}\right)_{\infty}}{\left(c, \frac{a}{c}, \frac{a}{c}, \frac{c q}{a}, \frac{b d}{a}, \frac{a q}{b d}, \frac{b e}{a}, \frac{a q}{b e}, \frac{b f q}{a}, \frac{a}{b f}, \frac{c d e f}{a^{2}}, \frac{a^{2} q}{c d e f}\right)_{\infty}}\right] \\
\times{ }_{8} W_{7}\left(\frac{b^{2}}{a} ; b, \frac{b c}{a}, \frac{b d}{a}, \frac{b e}{a}, \frac{b f}{a} ; \frac{a^{2} q^{2}}{b c d e f}\right) .
\end{aligned}
$$

We may derive (5.8) from the standard ${ }_{8} \phi_{7}$ transformation as follows. Starting from [7, (III. 37), p. 246], we first apply [7, (III. 23), p. 243] to one of the ${ }_{8} \phi_{7}$ 's in that formula, replacing

$$
{ }_{8} W_{7}\left(\frac{e f}{c} ; \frac{a q}{b c}, \frac{a q}{c d}, \frac{e f}{a}, e, f ; \frac{b d}{a}\right)
$$

by

$$
\frac{\left(\frac{e f q}{c}, \frac{b c d e f}{a^{2} q}, \frac{b q}{c}, \frac{d q}{c}\right)_{\infty}}{\left(\frac{b e f}{a}, \frac{d e f}{a}, \frac{a q^{2}}{c^{2}}, \frac{b d}{a}\right)_{\infty}} W_{7}\left(\frac{a q}{c^{2}} ; \frac{a q}{c f}, \frac{a q}{c e}, \frac{q}{c}, \frac{a q}{c b}, \frac{a q}{c d} ; \frac{b c d e f}{a^{2} q}\right) .
$$

If we now iterate the formula in the modified form and make use of Slater's infinite product identity [27], [7, p. 138], we can reduce the result to (5.8).

The standard three-term ${ }_{8} \phi_{7}$ formula easily provides a connection between the solutions $Y_{n}^{(1)}, Y_{n}^{(3)}$ and $Y_{n}^{(5)}$, and we actually have

$$
\begin{aligned}
\left(\frac{b^{2} q}{a}, a q\right. & \left.\frac{a}{b}, \frac{b q}{a}, \frac{a q}{c d}, \frac{a q}{c e}, \frac{a q}{d e}, \frac{b c d}{a}, \frac{b c e}{a}, \frac{b d e}{a}, \frac{c d e}{a}\right)_{\infty} Y_{n}^{(3)} \\
= & \left(a q, c, d, e, \frac{b q}{a}, \frac{b q}{c}, \frac{b q}{d}, \frac{b q}{e}, \frac{b c d e}{a}, \frac{b c d e}{a^{2}}, \frac{a^{2} q}{b c d e}\right)_{\infty} Y_{n}^{(5)} \\
& \quad-\frac{a}{b}\left(\frac{b^{2} q}{a}, \frac{a q}{b}, \frac{a q}{c}, \frac{a q}{d}, \frac{a q}{e}, \frac{b c}{a}, \frac{b d}{a}, \frac{b e}{a}, \frac{c d e}{a}, \frac{a q}{c d e}, \frac{b c d e}{a}\right)_{\infty} Y_{n}^{(1)} .
\end{aligned}
$$

On the other hand, (5.8) is better suited to provide a connection between the solutions $Y_{n}^{(1)}, Y_{n}^{(2)}$ and $Y_{n}^{(5)}$, and they are related by

$$
Y_{n}^{(1)}=R Y_{n}^{(2)}+S Y_{n}^{(5)},
$$


where

$$
\begin{aligned}
R= & -\frac{b d e h}{a^{2}} \frac{\left(a q, b, \frac{c}{a}, \frac{b q}{a}, \frac{d q}{a}, \frac{e q}{a}, \frac{a q}{c d}, \frac{a q}{c e}, \frac{a q}{d e}, \frac{a}{b}, \frac{b q}{a}\right)_{\infty}}{\left(\frac{q}{c}, \frac{q}{d}, \frac{q}{e}, \frac{a}{c}, \frac{a q}{b}, \frac{a q}{d}, \frac{a q}{e}, \frac{b c}{a}, \frac{b d}{a}, \frac{b e}{a}, \frac{q^{2}}{a}\right)_{\infty}} \frac{\left(\frac{c c d e h}{a^{2}}, \frac{a^{2} q}{b c d e h}, \frac{a}{q h}, \frac{h q^{2}}{a}\right)_{\infty}}{\left(\frac{c d e h}{a^{2}}, \frac{a^{2} q}{c d e h}, \frac{a}{b h}, \frac{b h q}{a}\right)_{\infty}}, \\
S= & \frac{\left(a q, c, \frac{c}{a}, \frac{b q}{a}, \frac{b q}{d}, \frac{b q}{e}, \frac{a q}{b d}, \frac{a q}{b e}\right)_{\infty}}{\left(\frac{b^{2} q}{a}, \frac{q}{d}, \frac{q}{e}, \frac{c}{b}, \frac{a q}{b}, \frac{a q}{d}, \frac{a q}{e}, \frac{b c}{a}\right)_{\infty}} \\
& \times\left[1-\frac{\left(b, \frac{q}{b}, \frac{a}{b}, \frac{b q}{a}, \frac{c d}{a}, \frac{a q}{c d}, \frac{c e}{a}, \frac{a q}{c e}, \frac{c h q}{a}, \frac{a}{c h}, \frac{b d e h}{a^{2}}, \frac{a^{2} q}{b d e h}\right)_{\infty}}{\left(c, \frac{q}{c}, \frac{a}{c}, \frac{c q}{a}, \frac{b d}{a}, \frac{a q}{b d}, \frac{b e}{a}, \frac{a q}{b e}, \frac{b h q}{a}, \frac{a}{b h}, \frac{c d e h}{a^{2}}, \frac{a^{2} q}{c d e h}\right)_{\infty}}\right] .
\end{aligned}
$$

We now give the continued fraction associated with the three-term recurrence (5.1). We follow the same procedure as in [12], where it was derived for the special case $h=1$. For the sake of completeness, the outline of the method and the related results are stated below.

In order to construct the minimal solution to (5.1) we examine the large $n$ asymptotics of the solutions $Y_{n}^{(1)}$ and $Y_{n}^{(3)}$. For $Y_{n}^{(1)}$ we first apply [7, (III. 23), p. 243] and then let $n \rightarrow \infty$ to obtain

$$
\begin{aligned}
Y_{n}^{(1)} & \approx(-1)^{n} C_{1}, \quad\left|\frac{a q}{d e}\right|<1, \\
C_{1} & =\frac{\left(a q, \frac{a q}{d e}\right)_{\infty}}{\left(\frac{a q}{d}, \frac{a q}{e}\right)_{\infty}} \phi_{2}\left(\frac{\frac{a q}{b c}, d, e}{\left.\frac{a q}{b}, \frac{a q}{c} ; \frac{a q}{d e}\right) .}\right.
\end{aligned}
$$

For $Y_{n}^{(3)}$, we apply [7, (III. 24), p. 243] and take the limit as $n \rightarrow \infty$. Subsequently using [7, (III. 9), p. 241], we have

$$
\begin{aligned}
Y_{n}^{(3)} & \approx(-1)^{n} C_{3}, \quad\left|\frac{b c}{a}\right|<1, \\
C_{3} & =\frac{\left(\frac{b c d e}{a}, \frac{b c}{a}\right)_{\infty}}{\left(\frac{b c d}{a}, \frac{b c e}{a}\right)_{\infty} \phi_{2}}\left(\begin{array}{l}
\frac{d e}{a}, d, e \\
\frac{c d e}{a}, \frac{b d e}{a}
\end{array} ; \frac{b e}{a}\right) .
\end{aligned}
$$

A minimal solution to (5.1) is now given by

$$
Y_{n}^{(\min )}=C_{3} Y_{n}^{(1)}-C_{1} Y_{n}^{(3)}
$$

Using Pincherle's theorem [8], [19] and simplifying, we have the continued fraction representation: 


\section{Theorem 5.1.}

$$
\begin{aligned}
& \frac{1}{c_{0}}-\frac{d_{1}}{c_{1}}-\frac{d_{2}}{c_{2}}-\cdots \\
& =\frac{Y_{0}^{(\min )}}{c_{0} Y_{0}^{(\min )}-Y_{1}^{(\min )}} \\
& =-\frac{1}{q\left(1-\frac{1}{h}\right)\left(1-\frac{a^{2} q}{b c d e h}\right)\left(1-\frac{a}{h}\right)} \\
& \times\left[C_{3}{ }_{8} W_{7}\left(a ; b, c, d, e, h ; \frac{a^{2} q^{2}}{b c d e h}\right)\right. \\
& \left.-C_{1} \frac{\left(\frac{b c d e}{a}, \frac{b c d e h}{a^{2} q}, \frac{b q}{h}, \frac{c q}{h}, \frac{d q}{h}, \frac{e q}{h}\right)_{\infty}}{\left(\frac{b d e}{a}, \frac{b c e}{a}, \frac{b c d}{a}, \frac{c d e}{a}, \frac{a q^{2}}{h^{2}}, \frac{q}{h}\right)_{\infty}}\left(\frac{a q}{h^{2}} ; \frac{a q}{b h}, \frac{a q}{c h}, \frac{a q}{d h}, \frac{a q}{e h}, \frac{q}{h} ; \frac{b c d e h}{a^{2} q}\right)\right] \\
& /\left[C_{3}{ }_{8} W_{7}\left(a ; b, c, d, e, h q ; \frac{a^{2} q}{b c d e h}\right)\right. \\
& \left.-C_{1} \frac{\left(\frac{b c d e}{a}, \frac{b c d e h}{a^{2}}, \frac{b}{h}, \frac{c}{h}, \frac{d}{h}, \frac{e}{h}\right)_{\infty}}{\left(\frac{b d e}{a}, \frac{b c e}{a}, \frac{b c d}{a}, \frac{c d e}{a}, \frac{a}{h^{2}}, \frac{1}{h}\right)_{\infty}} W_{7}\left(\frac{a}{h^{2} q} ; \frac{a}{b h}, \frac{a}{c h}, \frac{a}{d h}, \frac{a}{e h}, \frac{1}{h} ; \frac{b c d e h}{a^{2}}\right)\right] .
\end{aligned}
$$

In the special case $h=1$, the above theorem gives (see [12]):

Corollary 5.2. If $c_{n}, d_{n}$ are given by (5.1) and $h=1$, we have the continued fraction representation

$$
\begin{aligned}
& \frac{1}{c_{0}}-\frac{d_{1}}{c_{1}}-\frac{d_{2}}{c_{2}}-\cdots \\
& =\frac{b c d e}{a^{2} q^{2}}\left\{\frac{(1-a q)}{(1-b)(1-c)(1-d)(1-e)}{ }^{8} W_{7}\left(a q ; q, \frac{a q}{b}, \frac{a q}{c}, \frac{a q}{d}, \frac{a q}{e} ; \frac{b c d e}{a^{2} q}\right)\right. \\
& \left.-\frac{\left(\frac{b c}{a}, \frac{a q}{d}, \frac{a q}{e}, \frac{b d e}{a}, \frac{c d e}{a}, q\right)_{\infty}}{\left(\frac{a q}{d e}, b, c, d, e, \frac{b c d e}{a^{2} q}\right)_{\infty}} \frac{{ }_{3} \phi_{2}\left(\begin{array}{c}
d e / a, d, e \\
c d e / a, b d e / a
\end{array} ; \frac{b c}{a}\right)}{3 \phi_{2}\left(\begin{array}{c}
a q / b c, d, e \\
a q / b, a q / c
\end{array} ; \frac{a q}{d e}\right)}\right\} .
\end{aligned}
$$

On the other hand, if we write $f=q^{-m}, s=\frac{a^{3} q^{3-m}}{b c d e}, h=1$ in (3.32) and take the limit as $m \rightarrow \infty$, we obtain the equivalent continued fraction representation [10]: 
Corollary 5.2'. Under the same conditions as Corollary 5.2 we have the equivalent representation

$$
\begin{aligned}
& \frac{1}{c_{0}}-\frac{d_{1}}{c_{1}}-\frac{d_{2}}{c_{2}}-\ldots \\
& =\frac{\left(1-\frac{a}{q}\right)}{q\left(1-\frac{a}{b}\right)\left(1-\frac{a}{c}\right)\left(1-\frac{a}{d}\right)\left(1-\frac{a}{e}\right)}\left[{ }_{8} W_{7}\left(\frac{q}{a} ; \frac{q}{b}, \frac{q}{c}, \frac{q}{d}, \frac{q}{e}, q ; \frac{b c d e}{a^{2} q}\right)-R\right] \text {, } \\
& R=\frac{\left(q, a, \frac{q^{2}}{a}, \frac{d e}{a}, \frac{d c}{a}, \frac{e c}{a}\right)_{\infty}}{\left(\frac{d q}{a}, \frac{e q}{a}, \frac{c q}{a}, \frac{d e c}{a q}, \frac{a q}{b}, b\right)_{\infty}}\left\{3 \phi_{2}\left(\begin{array}{l}
\frac{q}{d}, \frac{q}{e}, \frac{q}{c} \\
\frac{q b}{a}, \frac{a q^{2}}{c d e}
\end{array} ;\right)\right.
\end{aligned}
$$

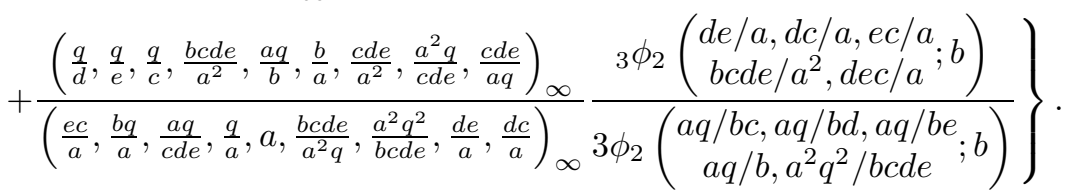

The above representation was obtained earlier in [10]. It is not obvious that this is the same as the representation (5.15). However, we can show that right sides of (5.15) and (5.16) are indeed equal. The proof involves use of the three-term ${ }_{8} \phi_{7}$ transformation formula (5.8), the ${ }_{3} \phi_{2}$ transformation [7, (III. 9), p. 241], the three-term ${ }_{3} \phi_{2}$ transformation formula [7, (III. 33), p. 245)] and Bailey's infinite product identity [7, Ex. 5.21, p. 138].

When the continued fraction terminates, we have a further simplification (see [12]):

Corollary 5.3. Under the conditions of Corollary (5.2) but with one of $\frac{a q}{b}, \frac{a q}{c}, \frac{a q}{d}$, or $\frac{a q}{e}$ equal to $q^{-N}, N=0,1, \ldots$, we have

$$
\begin{aligned}
& \frac{1}{c_{0}}-\frac{d_{1}}{c_{1}}-\frac{d_{2}}{c_{2}}-\cdots-\frac{d_{N}}{c_{N}} \\
& =\frac{b c d e}{a^{2} q^{2}} \frac{(1-a q)}{(1-b)(1-c)(1-d)(1-e)}{ }_{8} W_{7}\left(a q ; q, \frac{a q}{b}, \frac{a q}{c}, \frac{a q}{d}, \frac{a q}{e} ; \frac{b c d e}{a^{2} q}\right) .
\end{aligned}
$$

It is this last continued fraction result which is associated with the rational biorthogonality given in Corollary 4.3 for the special case $e=a q^{N+1}$ [12].

\section{THREE-TERM $\Phi$ TRANSFORMATION FORMULA}

Any three solutions out of the fifty-six solutions obtained for the second-order finite difference equation (3.2) in Section 3 are connected by a three-term $\Phi$ transformation formula. In order to derive such a formula, we start by picking any three of the solutions and assume a linear dependence. Here we consider

$$
X_{n}^{(1), \frac{s}{h} q^{n-1}}=P X_{n}^{(2), \frac{q^{n+1}}{h}}+Q X_{n}^{(3), \frac{b s}{a h} q^{n-1}}
$$


where $P$ and $Q$ are independent of $n$. If we take the large- $n$ asymptotics of (6.1), then from (3.20), (3.23) and (3.24) we obtain

$$
\begin{aligned}
{ }_{8} W_{7}\left(a ; b, c, d, e, f ; \frac{s}{a q}\right) \\
=P_{8} W_{7}\left(\frac{q}{a} ; \frac{q}{b}, \frac{q}{c}, \frac{q}{d}, \frac{q}{e}, \frac{q}{f} ; \frac{a q^{2}}{s}\right) \\
\quad+Q \frac{\left(\frac{s}{a q}, \frac{b q}{c}, \frac{b q}{d}, \frac{b q}{e}, \frac{b q}{f}, \frac{b q}{a}, \frac{b h q}{s}, \frac{s}{b h}\right)_{\infty}}{\left(\frac{b^{2} q}{a}, \frac{b c}{a}, \frac{b d}{a}, \frac{b e}{a}, \frac{b f}{a}, b, \frac{b h}{a}, \frac{a q}{b h}\right)_{\infty}\left(\frac{b^{2}}{a} ; b, \frac{b c}{a}, \frac{b d}{a}, \frac{b e}{a}, \frac{b f}{a} ; \frac{s}{a q}\right) .}
\end{aligned}
$$

Therefore from (5.8)

$$
P=\frac{\left(a q, b, \frac{b}{a}, \frac{b q}{a}, \frac{c q}{a}, \frac{d q}{a}, \frac{e q}{a}, \frac{f q}{a}, \frac{a q}{c d}, \frac{a q}{c e}, \frac{a q}{c f}\right)_{\infty}}{\left.\left(\frac{q^{2}}{a}, \frac{q}{c}, \frac{q}{d}, \frac{q}{e}, \frac{q}{f}, \frac{a q}{c}, \frac{a q}{d e}, \frac{a q}{e}, \frac{a q}{f}, \frac{b c}{a f}, \frac{b d}{a}\right)_{\infty}, \frac{a q}{e f}, \frac{b c d e f}{a^{2} q}\right)_{\infty}}
$$

and

$$
\begin{aligned}
Q=\frac{\left(a q, b, c, \frac{c}{a}, \frac{b d}{a}, \frac{a q}{b d}, \frac{b e}{a}, \frac{a q}{b e}, \frac{b f}{a}, \frac{a q}{b f}, \frac{b h}{a}, \frac{a q}{b h}\right)_{\infty}}{\left(\frac{s}{a q}, \frac{q}{d}, \frac{q}{e}, \frac{q}{f}, \frac{a q}{b}, \frac{a q}{d}, \frac{a q}{e}, \frac{a q}{f}, \frac{c}{b}, \frac{b q}{c}, \frac{s}{b h}, \frac{b h q}{s}\right)_{\infty}} \\
\quad \times\left[1-\frac{\left(b, \frac{q}{b}, \frac{a}{b}, \frac{b q}{a}, \frac{c d}{a}, \frac{a q}{c d}, \frac{c e}{a}, \frac{a q}{c e}, \frac{c f q}{a}, \frac{a}{c f}, \frac{b d e f}{a^{2}}, \frac{a^{2} q}{b d e f}\right)_{\infty}}{\left.\left(c, \frac{q}{c}, \frac{a}{c}, \frac{c q}{a}, \frac{b d}{a}, \frac{a q}{b d}, \frac{b e}{a}, \frac{a q}{b e}, \frac{b f q}{a}, \frac{a}{b f}, \frac{c d e f}{a^{2}}, \frac{a^{2} q}{c d e f}\right)_{\infty}\right] .}\right.
\end{aligned}
$$

We now substitute these $P$ and $Q$ in (6.1) and replace $\left(a, b, c, d, e, f, \frac{s}{h} q^{n-1}, h q^{-n}\right)$ by $(A, B, C, D, E, F, G, H)$ respectively. The result can be written as a three-term $\Phi$ transformation formula:

$$
\begin{aligned}
& \Phi^{(G)}(A ; B, C, D, E, F, G, H ; q) \\
& =\frac{\left(A q, B, G, \frac{B}{A}, \frac{G}{A}, \frac{B q}{A}, \frac{C q}{A}, \frac{D q}{A}, \frac{E q}{A}, \frac{F q}{A}, \frac{G q}{A}\right)_{\infty}}{\left(\frac{q^{2}}{A}, \frac{q}{C}, \frac{q}{D}, \frac{q}{E}, \frac{q}{F}, \frac{q}{H}, \frac{A}{H}, \frac{A q}{C}, \frac{A q}{D}, \frac{A q}{E}, \frac{A q}{F}, \frac{A q}{H}\right)_{\infty}} \\
& \times \frac{\left(\frac{A q}{C D}, \frac{A q}{C E}, \frac{A q}{C F}, \frac{A q}{D E}, \frac{A q}{D F}, \frac{A q}{E F}, \frac{A q}{B H}, \frac{A q}{C H}, \frac{A q}{D H}, \frac{A q}{E H}, \frac{A q}{F H}, \frac{A q}{G H}\right)_{\infty}}{\left(\frac{B C}{A}, \frac{B D}{A}, \frac{B E}{A}, \frac{B F}{A}, \frac{B G}{A}, \frac{C G}{A}, \frac{D G}{A}, \frac{E G}{A}, \frac{F G}{A}, \frac{C D E F}{A^{2}}, \frac{A^{2} q}{C D E F}\right)_{\infty}} \\
& \times \Phi^{\left(\frac{q}{H}\right)}\left(\frac{q}{A} ; \frac{q}{B}, \frac{q}{C}, \frac{q}{D}, \frac{q}{E}, \frac{q}{F}, \frac{q}{G}, \frac{q}{H} ; q\right) \\
& +\frac{\left(A q, B, C, \frac{C}{A}, \frac{G}{A}, \frac{G q}{A}, \frac{G q}{C}, \frac{G q}{D}, \frac{G q}{E}, \frac{G q}{F}, \frac{G q}{H}\right)_{\infty}}{\left(\frac{G^{2} q}{A}, \frac{q}{D}, \frac{q}{E}, \frac{q}{F}, \frac{q}{H}, \frac{A q}{B}, \frac{A q}{D}, \frac{A q}{E}, \frac{A q}{F}, \frac{A q}{H}, \frac{B}{G}\right)_{\infty}} \\
& \times \frac{\left(\frac{B D}{A}, \frac{A q}{B D}, \frac{B E}{A}, \frac{A q}{B E}, \frac{B F}{A}, \frac{A q}{B F}, \frac{B H}{A}, \frac{A q}{B H}\right)_{\infty}}{\left(\frac{C}{B}, \frac{B q}{C}, \frac{G B}{A}, \frac{G C}{A}, \frac{G D}{A}, \frac{G E}{A}, \frac{G F}{A}, \frac{G H}{A}\right)_{\infty}} \\
& \times\left[1-\frac{\left(B, \frac{q}{B}, \frac{A}{B}, \frac{B q}{A}, \frac{C D}{A}, \frac{A q}{C D}, \frac{C E}{A}, \frac{A q}{C E}, \frac{C F q}{A}, \frac{A}{C F}, \frac{B D E F}{A^{2}}, \frac{A^{2} q}{B D E F}\right)_{\infty}}{\left(C, \frac{q}{C}, \frac{A}{C}, \frac{C q}{A}, \frac{B D}{A}, \frac{A q}{B D}, \frac{B E}{A}, \frac{A q}{B E}, \frac{B F q}{A}, \frac{A}{B F}, \frac{C D E F}{A^{2}}, \frac{A^{2} q}{C D E F}\right)_{\infty}}\right] \\
& \times \Phi^{\left(\frac{B G}{A}\right)}\left(\frac{G^{2}}{A} ; \frac{G B}{A}, \frac{G C}{A}, \frac{G D}{A}, \frac{G E}{A}, \frac{G F}{A}, G, \frac{G H}{A} ; q\right) \text {. }
\end{aligned}
$$




\section{Particular cases.}

1. In the general formula, if we write $B=q^{-n}$, then it reduces to the terminating ${ }_{10} \phi_{9}$ transformation

$$
\begin{aligned}
& { }_{10} W_{9}\left(A ; C, D, E, F, G, H, q^{-n} ; q\right) \\
& =\frac{\left(A q, C, G, \frac{C}{A}, \frac{G}{A}, \frac{A q^{n+1}}{D}, \frac{A q^{n+1}}{E}, \frac{A q^{n+1}}{F}, \frac{A q^{n+1}}{H}, \frac{q^{-n+1}}{D}, \frac{q^{-n+1}}{E}, \frac{q^{-n+1}}{F}, \frac{q^{-n+1}}{H}, \frac{q^{-n+1}}{A}\right)_{\infty}}{\left(\frac{q^{-2 n+1}}{A}, \frac{q}{D}, \frac{q}{E}, \frac{q}{F}, \frac{q}{H}, \frac{A q}{D}, \frac{A q}{E}, \frac{A q}{F}, \frac{A q}{H}, A q^{n+1}, C q^{n}, G q^{n}, \frac{C}{A} q^{-n}, \frac{G}{A} q^{-n}\right)_{\infty}} \\
& \quad \times{ }_{10} W_{9}\left(\frac{q^{-2 n}}{A} ; \frac{C q^{-n}}{A}, \frac{D q^{-n}}{A}, \frac{E q^{-n}}{A}, \frac{F q^{-n}}{A}, \frac{G q^{-n}}{A}, \frac{H q^{-n}}{A}, q^{-n} ; q\right) .
\end{aligned}
$$

The above formula can be derived also with the help of known formulas. Refer to the terminating ${ }_{10} \phi_{9}$ transformation formula [7, Ex. 2.19, p. 53] to which, if we apply the ${ }_{10} \phi_{9}$ transformation formula [7, Ex. 2.30, p. 56], we obtain (6.6).

2. Writing $G=q^{-n}$ in (6.5) we obtain another relation between two terminating ${ }_{10} \phi_{9}$ 's as in the above paragraph. For the particular value $n=0$, the relation reduces to Slater's infinite product identity ([27]; also see [7, Ex. 5.22, p. 138].

3. In the general formula (6.5) we interchange the parameters $B \leftrightarrow C$ and then eliminate $\Phi^{\left(\frac{q}{H}\right)}\left(\frac{q}{A} ; \frac{q}{B}, \frac{q}{C}, \frac{q}{D}, \frac{q}{E}, \frac{q}{F}, \frac{q}{G}, \frac{q}{H} ; q\right)$ from the two equations. Using Slater's infinite product identity we can write the result in the following form:

$$
\begin{aligned}
& \frac{\left(\frac{A q}{D}, \frac{A q}{E}, \frac{A q}{F}, \frac{A q}{H}, \frac{q}{D}, \frac{q}{E}, \frac{q}{F}, \frac{q}{H}\right)_{\infty}}{\left(A q, B, C, G, \frac{B}{A}, \frac{C}{A}, \frac{G}{A}\right)_{\infty}}{ }_{10} W_{9}(A ; B, C, \ldots, H ; q) \\
& =-\frac{A}{B} \frac{\left(\frac{B q}{D}, \frac{B q}{E}, \frac{B q}{F}, \frac{B q}{H}, \frac{A q}{B D}, \frac{A q}{B E}, \frac{A q}{B F}, \frac{A q}{B H}\right)_{\infty}}{\left(\frac{B^{2} q}{A}, B, \frac{B C}{A}, \frac{B G}{A}, \frac{A}{B}, \frac{C}{B}, \frac{G}{B}\right)_{\infty}}\left(\frac{B^{2}}{A} ; B, \frac{B C}{A}, \frac{B D}{A}, \ldots \frac{B H}{A} ; q\right) \\
& +i \operatorname{dem}(B ; C, G) \text {. }
\end{aligned}
$$

The above is a particular case of $[7$, Ex. 4.6, p. 122] when the balance condition is satisfied. Slater has obtained (6.7) from other considerations and then used it to derive her infinite product identity. However, we have shown here that, from our general formula, we can derive Slater's infinite product identity and consequently also (6.7).

4. In (6.5), if we take the value $\frac{B G}{A}=1$ and use Slater's infinite product identity we can reduce the result to Bailey's non-terminating extension of Jackson's ${ }_{8} \phi_{7} \operatorname{sum}[7$, (II. 25), p. 238].

Limiting cases. All the known ${ }_{8} \phi_{7}$ two-term and three-term transformation formulas may be obtained as limiting cases of our general transformation formula (6.5). When we let one of the parameters in the $\Phi(A ; B, C, D, E, F, G, H ; q)$ tend to 0 and another tend to infinity so that the balance condition remains intact, we obtain in general a relation connecting three, four or five ${ }_{8} \phi_{7}$ 's depending on the choice of parameters. The ${ }_{8} \phi_{7}$ series involved are

$$
{ }_{8} W_{7}\left(A ; B, C, D, E, F ; \frac{A^{2} q^{2}}{B C D E F}\right),
$$


its reflection

$$
{ }_{8} W_{7}\left(\frac{q}{A} ; \frac{q}{B}, \frac{q}{C}, \frac{q}{D}, \frac{q}{E}, \frac{q}{F} ; \frac{B C D E F}{A^{2} q}\right),
$$

and complements of both, i.e., series of the type

$$
{ }_{8} W_{7}\left(\frac{B^{2}}{A} ; \frac{B C}{A}, \frac{B D}{A}, \frac{B E}{A}, \frac{B F}{A}, B ; \frac{A^{2} q^{2}}{B C D E F}\right)
$$

and

$$
{ }_{8} W_{7}\left(\frac{A q}{B^{2}} ; \frac{A q}{B C}, \frac{A q}{B D}, \frac{A q}{B E}, \frac{A q}{B F}, \frac{q}{B} ; \frac{B C D E F}{A^{2} q}\right) .
$$

We give below some of the interesting limiting cases.

1. Replace $B$ and $G$ by $b q^{-m}$ and $g q^{m}$ respectively, and let $m \rightarrow \infty$. We arrive at a relation connecting five ${ }_{8} \phi_{7}$ 's. However, if we write $b=1$ in this relation after using the balance condition to eliminate $g$, we just obtain the transformation formula [7, (III. 24), p. 243] of a very-well-poised ${ }_{8} \phi_{7}$ series into another very-well-poised ${ }_{8} \phi_{7}$-series.

2. Replacing $B$ and $H$ by $b q^{-m}$ and $h q^{m}$ respectively and letting $m \rightarrow \infty$ leads in general to a representation of an $8 \phi_{7}$ as sum of $a_{4} \phi_{3}$ and a ${ }_{4} \psi_{4}$. Using now the balance condition to eliminate $h$ and then writing the special value $b=1$ yields the following representation of an ${ }_{8} \phi_{7}$ as a sum of two ${ }_{4} \phi_{3}$ 's:

$$
\begin{aligned}
& { }_{8} W_{7}\left(A ; C, D, E, F, G ; \frac{A^{2} q^{2}}{C D E F G}\right) \\
& =\frac{\left(A q, E, \frac{A q}{C G}, \frac{A q}{D G}, \frac{A q}{F G}, \frac{A^{2} q^{2}}{C D E F}\right)_{\infty}}{\left(\frac{A q}{C}, \frac{A q}{D}, \frac{A q}{F}, \frac{A q}{G}, \frac{E}{G}, \frac{A^{2} q^{2}}{C D E F G}\right)_{\infty}} \phi_{3}\left(\begin{array}{l}
\frac{A q}{C E}, \frac{A q}{D E}, \frac{A q}{F E}, G \\
\frac{A q}{E}, \frac{G q}{E}, \frac{A^{2} q^{2}}{C D E F} ; q
\end{array}\right) \\
& +\frac{\left(A q, G, \frac{A q}{C E}, \frac{A q}{D E}, \frac{A q}{F E}, \frac{A^{2} q^{2}}{C D F G}\right)_{\infty}}{\left(\frac{A q}{C}, \frac{A q}{D}, \frac{A q}{E}, \frac{A q}{F}, \frac{G}{E}, \frac{A^{2} q^{2}}{C D E F G}\right)_{\infty}} \phi_{3}\left(\begin{array}{l}
\frac{A q}{C G}, \frac{A q}{D G}, \frac{A q}{F G}, E \\
\frac{A q}{G}, \frac{E q}{G}, \frac{A^{2} q^{2}}{C D F G} ; q
\end{array}\right) .
\end{aligned}
$$

The above is equivalent to the standard three-term transformation formula [7, (III. 36), p. 246].

3. Replace $E$ and $F$ by $e q^{-m}$ and $f q^{m}$ respectively and take the limit as $m \rightarrow \infty$. The general formula reduces to a relation connecting five ${ }_{8} \phi_{7}$ 's. For particular values of the parameters this relation reduces to some known transformation formulas.

A first particular case of the above is obtained by eliminating $f$ with the help of the balance condition and then writing $e=1, B=q^{-n}$. The result reduces to Sear's transformation of terminating balanced ${ }_{4} \phi_{3}$ series [7, (III. 15), p. 242].

A second particular case is obtained as follows. After eliminating $f$ as before, we take the special value $e=\frac{A^{2} q^{2}}{B D G H}$. The resulting relation connects the three ${ }_{8} \phi_{7}$ 's which are complement to each other viz.,

$$
\begin{aligned}
& { }_{8} W_{7}\left(A ; B, C, D, G, H ; \frac{A^{2} q^{2}}{B C D G H}\right), \\
& { }_{8} W_{7}\left(\frac{B^{2}}{A} ; B, \frac{B C}{A}, \frac{B D}{A}, \frac{B G}{A}, \frac{B H}{A} ; \frac{A^{2} q^{2}}{B C D G H}\right), \\
& { }_{8} W_{7}\left(\frac{G^{2}}{A} ; G, \frac{G B}{A}, \frac{G C}{A}, \frac{G D}{A}, \frac{G H}{A} ; \frac{A^{2} q^{2}}{B C D G H}\right) .
\end{aligned}
$$


An application of Bailey's infinite product identity [7, Ex. 5.21, p. 138] enables us to write the above transformation formula in the form given by Bailey [3] (also refer to [7, Ex. 2.15, p. 52].

4. Write $G=g q^{m}, H=h q^{-m}$ in (6.5) and take the limit as $m \rightarrow \infty$. This results in the three-term ${ }_{8} \phi_{7}$ transformation (5.8).

\section{QuAdRATIC IDENTITIES}

Any two linearly independent solutions $X_{n}^{(i)}$ and $X_{n}^{(j)}$ of the three-term recurrence (3.2) satisfy the formula

$$
\lim _{n \rightarrow \infty} \frac{\mathcal{W}\left(X_{n}^{(i)}, X_{n}^{(j)}\right)}{b_{1} b_{2} \ldots b_{n}}=b_{0} \mathcal{W}\left(X_{-1}^{(i)}, X_{-1}^{(j)}\right)
$$

where the Casorati determinant

$$
\mathcal{W}\left(X_{n}^{(i)}, X_{n}^{(j)}\right):=X_{n}^{(i)} X_{n+1}^{(j)}-X_{n+1}^{(i)} X_{n}^{(j)} .
$$

Taking second order asymptotics of (7.1), we may derive quadratic identities involving ${ }_{8} \phi_{7}$ 's. We demonstrate this below by considering pairs of solutions from the solutions $X_{n}^{(1), \frac{s q^{n-1}}{h}}, X_{n}^{(2), \frac{q^{n+1}}{h}}$ and $X_{n}^{(3), \frac{b s}{a h} q^{n-1}}$.

If $n, k$ are non-negative integers and $\alpha, \beta$ are independent of $n$, then

$$
\begin{aligned}
\left(\alpha q^{-n}\right)_{k} & =\left(1-a_{1} \frac{q^{n}}{\alpha}+a_{2} \frac{q^{2 n}}{\alpha^{2}}+O\left(q^{3 n}\right)\right)(-\alpha)^{k} q^{k(k-1) / 2-n k}, \\
\left(\beta q^{n}\right)_{k} & =1-b_{1} \beta q^{n}+b_{2} \beta^{2} q^{2 n}+O\left(q^{3 n}\right),
\end{aligned}
$$

where

$$
\begin{aligned}
& a_{1}=q^{-k+1} \frac{\left(1-q^{k}\right)}{(1-q)}, \quad a_{2}=q^{-k+1} q^{-k+2} \frac{\left(1-q^{k-1}\right)\left(1-q^{k}\right)}{(1-q)\left(1-q^{2}\right)}, \\
& b_{1}=\frac{\left(1-q^{k}\right)}{(1-q)}, \quad b_{2}=\frac{q\left(1-q^{k-1}\right)\left(1-q^{k}\right)}{(1-q)\left(1-q^{2}\right)} .
\end{aligned}
$$

Using (7.2), we can work out the second order large $n$ asymptotics of the three solutions mentioned above. Starting from (3.3), we obtain

$$
\begin{aligned}
& X_{n}^{(1), \frac{s}{h} q^{n-1}} \approx W_{1}-\frac{q^{n}}{h} T_{1} W_{1+}+\frac{q^{n}}{h} S_{1} W_{1}+O\left(q^{2 n}\right), \\
& W_{1}={ }_{8} W_{7}\left(a ; b, c, d, e, f ; \frac{s}{a q}\right), \\
& W_{1+}={ }_{8} W_{7}\left(a q^{2} ; b q, c q, d q, e q, f q ; \frac{s}{a q^{2}}\right), \\
& S_{1}=\frac{1}{(1-q)}\left(\frac{s}{q}+\frac{s}{a q}+\frac{a q}{b}+\frac{a q}{c}+\frac{a q}{d}+\frac{a q}{e}+\frac{a q}{f}-a q\right), \\
& T_{1}=\frac{\frac{s}{a q}\left(1-\frac{s}{a q^{2}}\right)(1-a q)\left(1-a q^{2}\right)(1-b)(1-c)(1-d)(1-e)(1-f)}{(1-q)\left(1-\frac{a q}{b}\right)\left(1-\frac{a q}{c}\right)\left(1-\frac{a q}{d}\right)\left(1-\frac{a q}{e}\right)\left(1-\frac{a q}{f}\right)} .
\end{aligned}
$$


In the above calculation we were surprised to find that not only the first but also the second order asymptotics were given in terms of very-well-poised ${ }_{8} \phi_{7}$ 's. We have not checked if this continues to hold in higher orders.

From (3.8) we obtain

$$
\begin{aligned}
& X_{n}^{(3), \frac{b s}{a h} q^{n-1}} \approx L_{3}\left(W_{3}-\frac{q^{n}}{h} T_{3} W_{3+}+\frac{q^{n}}{h} S_{3} W_{3}\right)+O\left(q^{2 n}\right), \\
& W_{3}={ }_{8} W_{7}\left(\frac{b^{2}}{a} ; b, \frac{b c}{a}, \frac{b d}{a}, \frac{b e}{a}, \frac{b f}{a} ; \frac{s}{a q}\right) \\
& W_{3+}={ }_{8} W_{7}\left(\frac{b^{2} q^{2}}{a} ; b q, \frac{b c q}{a}, \frac{b d q}{a}, \frac{b e q}{a}, \frac{b f q}{a} ; \frac{s}{a q^{2}}\right), \\
& S_{3}=(1-q)^{-1}\left(\frac{a q}{c}+\frac{a q}{d}+\frac{a q}{e}+\frac{a q}{f}+\frac{b s}{a q}+q+\frac{s}{b q}-b q\right), \\
& T_{3}=\frac{\frac{s}{b q}\left(1-\frac{s}{a q^{2}}\right)\left(1-\frac{b^{2} q}{a}\right)\left(1-\frac{b^{2} q^{2}}{a}\right)\left(1-\frac{b c}{a}\right)\left(1-\frac{b d}{a}\right)\left(1-\frac{b e}{a}\right)\left(1-\frac{b f}{a}\right)(1-b)}{(1-q)\left(1-\frac{b q}{c}\right)\left(1-\frac{b q}{d}\right)\left(1-\frac{b q}{e}\right)\left(1-\frac{b q}{f}\right)\left(1-\frac{b q}{a}\right)}, \\
& L_{3}=\frac{\left(\frac{s}{a q}, \frac{b q}{c}, \frac{b q}{d}, \frac{b q}{e}, \frac{b q}{f}, \frac{b q}{a}, \frac{b h q}{s}, \frac{s}{b h}\right)_{\infty}}{\left(\frac{b^{2} q}{a}, \frac{b c}{a}, \frac{b d}{a}, \frac{b e}{a}, \frac{b f}{a}, b, \frac{b h}{a}, \frac{a q}{b h}\right)_{\infty}}
\end{aligned}
$$

From (3.6) we have

$$
\begin{aligned}
& X_{n}^{(2), \frac{q^{n+1}}{h}} \approx W_{2}-\frac{q^{n}}{h} T_{2} W_{2+}+\frac{q^{2}}{h} S_{2} W_{2}+O\left(q^{2 n}\right), \\
& W_{2}={ }_{8} W_{7}\left(\frac{q}{a} ; \frac{q}{b}, \frac{q}{c}, \frac{q}{d}, \frac{q}{e}, \frac{q}{f} ; \frac{a q^{2}}{s}\right), \\
& W_{2+}={ }_{8} W_{7}\left(\frac{q^{3}}{a} ; \frac{q^{2}}{b}, \frac{q^{2}}{c}, \frac{q^{2}}{d}, \frac{q^{2}}{e}, \frac{q^{2}}{f} ; \frac{a q}{s}\right), \\
& S_{2}=\frac{1}{(1-q)}\left(q+a+\frac{b s}{a q}+\frac{c s}{a q}+\frac{d s}{a q}+\frac{e s}{a q}+\frac{f s}{a q}-\frac{s}{a}\right), \\
& T_{2}=\frac{a\left(1-\frac{a q}{s}\right)\left(1-\frac{q^{2}}{a}\right)\left(1-\frac{q^{3}}{a}\right)\left(1-\frac{q}{b}\right)\left(1-\frac{q}{c}\right)\left(1-\frac{q}{d}\right)\left(1-\frac{q}{e}\right)\left(1-\frac{q}{f}\right)}{(1-q)\left(1-\frac{b q}{a}\right)\left(1-\frac{c q}{a}\right)\left(1-\frac{d q}{a}\right)\left(1-\frac{e q}{a}\right)\left(1-\frac{f q}{a}\right)} .
\end{aligned}
$$

Looking at the convergence conditions of the different ${ }_{8} \phi_{7}$ series in (7.3), (7.4) and (7.5), we find that the arguments of series in (7.5) are not compatible with those of (7.3) and (7.4). The difficulty can be overcome by applying the transformation [7, (III. 39), p. 247] to the $\Phi$ in $X_{n}^{(2), \frac{q^{n+1}}{h}}$ before working out its asymptotics. We 
then obtain

$$
\begin{aligned}
& X_{n}^{(2), \frac{q^{n+1}}{h}} \approx L_{2}^{\prime}\left(W_{2}^{\prime}-\frac{q^{n}}{h} T_{2}^{\prime} W_{2+}^{\prime}+\frac{q^{n}}{h} S_{2}^{\prime} W_{2}^{\prime}\right)+O\left(q^{2 n}\right), \\
& W_{2}^{\prime}={ }_{8} W_{7}\left(\frac{c d e}{a^{2}} ; \frac{c e}{a}, \frac{c d}{a}, \frac{d e}{a}, \frac{q}{f}, \frac{q}{b} ; \frac{b f}{a}\right), \\
& W_{2+}^{\prime}={ }_{8} W_{7}\left(\frac{c d e q^{2}}{a^{2}} ; \frac{c e q}{a}, \frac{c d q}{a}, \frac{d e q}{a}, \frac{q^{2}}{f}, \frac{q^{2}}{b} ; \frac{b f}{a q}\right), \\
& S_{2}^{\prime}=\frac{1}{(1-q)}\left(q+\frac{c s}{a q}+\frac{d s}{a q}+\frac{e s}{a q}+\frac{a^{2} q}{c d e}+\frac{a q}{f}+\frac{a q}{b}-\frac{a q^{2}}{b f}\right), \\
& T_{2}^{\prime}=\frac{\frac{b f s}{a q^{2}}\left(1-\frac{b f}{a q}\right)\left(1-\frac{c d e q}{a^{2}}\right)\left(1-\frac{c d e q^{2}}{a^{2}}\right)\left(1-\frac{c d}{a}\right)\left(1-\frac{c e}{a}\right)\left(1-\frac{d e}{a}\right)\left(1-\frac{q}{f}\right)\left(1-\frac{q}{b}\right)}{(1-q)\left(1-\frac{c q}{a}\right)\left(1-\frac{d q}{a}\right)\left(1-\frac{e q}{a}\right)\left(1-\frac{c d e f}{a^{2}}\right)\left(1-\frac{b c d e}{a^{2}}\right)}, \\
& L_{2}^{\prime}=\frac{\left(\frac{q^{2}}{a}, \frac{c d e f}{a^{2}}, \frac{b c d e}{a^{2}}, \frac{b f}{a}\right)_{\infty}}{\left(\frac{c d d e q}{a^{2}}, \frac{f q}{a}, \frac{b q}{a}, \frac{a q^{2}}{s}\right)_{\infty}}
\end{aligned}
$$

We now apply (7.1) to pairs of solutions from $X_{n}^{(1), \frac{s}{h} q^{n-1}}, X_{n}^{(2), \frac{q^{n+1}}{h}}$ and $X_{n}^{(3), \frac{b s}{a h} q^{n-1}}$. In the particular case $h=1$, the right side of (7.1) can be evaluated in terms of infinite products. Thus we obtain the three identities

$$
\begin{aligned}
& L_{2}^{\prime}(1-q)[\left.\left(S_{1}-S_{2}^{\prime}\right) W_{1} W_{2}^{\prime}-T_{1} W_{1+} W_{2}^{\prime}+T_{2}^{\prime} W_{1} W_{2+}^{\prime}\right] \\
&=- q\left(1-\frac{a}{b}\right)\left(1-\frac{a}{c}\right)\left(1-\frac{a}{d}\right)\left(1-\frac{a}{e}\right)\left(1-\frac{a}{f}\right) \frac{(a q)_{\infty}}{\left(\frac{a}{q}\right)_{\infty}} \\
& \times {\left[1+\frac{\left(\frac{a}{q}, \frac{q^{2}}{a}, b, \frac{q}{b}, c, \frac{q}{c}, d, \frac{q}{d}, e, \frac{q}{e}, f, \frac{q}{f}, \frac{s}{q^{2}}, \frac{q^{3}}{s}\right)_{\infty}}{\left(a, \frac{q}{a}, \frac{a}{b}, \frac{b q}{a}, \frac{a}{c}, \frac{c q}{a}, \frac{a}{d}, \frac{d q}{a}, \frac{a}{e}, \frac{e q}{a}, \frac{a}{f}, \frac{f q}{a}, \frac{s}{a q}, \frac{a q^{2}}{s}\right)_{\infty}}\right], } \\
& L_{3}^{\prime}(1-q)\left[\left(S_{1}-S_{3}\right) W_{1} W_{3}-T_{1} W_{1+} W_{3}+T_{3} W_{1} W_{3+}\right] \\
&=-\frac{a q^{2}}{s} \frac{\left(a q, \frac{s}{b q}, \frac{b q^{2}}{s}, \frac{a q}{d e}, \frac{a q}{c e}, \frac{a q}{c d}, \frac{a q}{c f}, \frac{a q}{d f}, \frac{a q}{e f}\right)_{\infty}}{\left(\frac{a q}{b}, \frac{a q}{c}, \frac{a q}{d}, \frac{a q}{e}, \frac{a q}{f}, \frac{b c}{a}, \frac{b d}{a}, \frac{b e}{a}, \frac{b f}{a}\right)_{\infty}}, \\
& L_{2}^{\prime} L_{3}^{\prime}(1-q)\left[\left(S_{2}^{\prime}-S_{3}\right) W_{2}^{\prime} W_{3}-T_{2}^{\prime} W_{2+}^{\prime} W_{3}+T_{3} W_{2}^{\prime} W_{3+}\right] \\
&=-\frac{a q^{2}}{s} \frac{\left(\frac{q^{2}}{a}, \frac{q}{c}, \frac{q}{d}, \frac{q}{e}, \frac{q}{f}, \frac{c d e f}{a^{2}}, \frac{a^{2} q}{c d e f}, \frac{s}{b q}, \frac{b q^{2}}{s}\right)_{\infty}}{\left(b, \frac{b}{a}, \frac{b q}{a}, \frac{c q}{a}, \frac{d q}{a}, \frac{e q}{a}, \frac{f q}{a}, \frac{a q}{b}, \frac{a q^{2}}{s}\right)_{\infty}},
\end{aligned}
$$

where $L_{3}^{\prime}$ is the $L_{3}$ of (7.4) with $h=1$.

The identities (7.7) and (7.8) are easy to derive. However, in the derivation of (7.9) we had to make use of the $\Phi$ transformation formula (6.5) for special values

$$
(A, B, C, D, E, F, G, H) \rightarrow\left(a, b, c, d, e, f, \frac{s}{q}, 1\right)
$$

and also Slater's infinite product identity.

It should be noted that these identities are not independent. Starting from any two of the three identities (7.7), (7.8) and (7.9), we can deduce the third one. For example, if we multiply $(7.7)$ by $\frac{W_{3}}{L_{2}^{\prime}(1-q)}$ and $(7.8)$ by $\frac{W_{3}}{L_{3}(1-q)}$ and subtract, we 
obtain the value of

$$
\left(S_{2}^{\prime}-S_{3}\right) W_{2}^{\prime} W_{3}-T_{2}^{\prime} W_{2+}^{\prime} W_{3}+T_{3} W_{2}^{\prime} W_{3+}
$$

in terms of infinite products and the $W_{1}, W_{2}^{\prime}, W_{3}$. If we now apply the three-term ${ }_{8} \phi_{7}$ transformation formula (5.8) and Slater's infinite product identity, we can write down the result in the form of identity (7.9).

We do not know where (7.7), (7.8) and (7.9) fit into the general scheme of $q$-series identities. In order to understand this better we now calculate a limiting case of (7.8) where $f=q^{-m}$ and $m \rightarrow \infty$.

If $\left|\frac{a q}{d e}\right|<1$ then, using [7, (III. 36), p. 246], we find that

$$
\lim _{m \rightarrow \infty}{ }_{8} W_{7}\left(a ; b, c, d, e, q^{-m} ; \frac{a^{2} q^{m+2}}{b c d e}\right)=\frac{\left(a q, \frac{a q}{d e}\right)_{\infty}}{\left(\frac{a q}{d}, \frac{a q}{e}\right)_{\infty}} \phi_{2}\left(\begin{array}{l}
\frac{a q}{b c}, d, e \\
\frac{a q}{b}, \frac{a q}{c}
\end{array} ; \frac{a q}{d e}\right),
$$

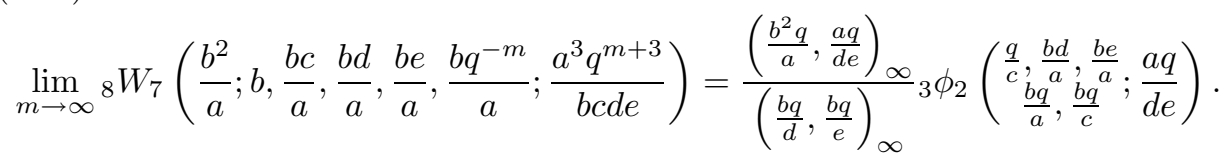

This gives the $f=q^{-m}, m \rightarrow \infty$ limit of the $W_{1}$ and $W_{3}$ in (7.8). We omit further details and now state the final result.

The $f=q^{-m}, m \rightarrow \infty$ limit of (7.8) yields

$$
\begin{aligned}
& { }_{3} \phi_{2}\left(\begin{array}{l}
\frac{a q}{b c}, d, e \\
\frac{a q}{b}, \frac{a q}{c} ; \frac{a q}{d e}
\end{array}\right){ }_{3} \phi_{2}\left(\begin{array}{l}
\frac{q}{c}, \frac{b d}{a}, \frac{b e}{a} \\
\frac{b q}{a}, \frac{b q}{c}
\end{array} \frac{a q}{d e}\right) \\
& +\frac{a q}{c d e} \frac{(1-c)(1-d)(1-e)}{\left(1-\frac{b}{a}\right)\left(1-\frac{a q}{b}\right)\left(1-\frac{a q}{c}\right)} 3 \phi_{2}\left(\begin{array}{l}
\frac{a q}{b c}, d q, e q \\
\frac{a q^{2}}{b}, \frac{a q^{2}}{c} ; \frac{a q}{d e}
\end{array}\right){ }_{3} \phi_{2}\left(\begin{array}{l}
\frac{q}{c}, \frac{b d}{a}, \frac{b e}{a} \\
\frac{b q}{a}, \frac{a q}{c}
\end{array} ; \frac{a q}{d e}\right)
\end{aligned}
$$

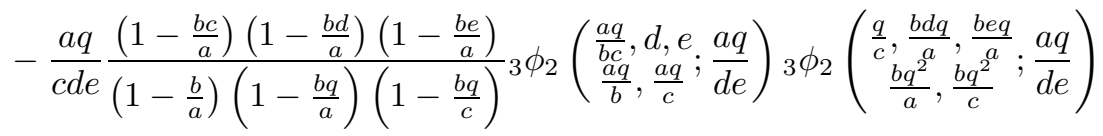

$$
\begin{aligned}
& =\frac{\left(\frac{a q}{c e}, \frac{a q}{c d}, b q\right)_{\infty}}{\left(\frac{a q}{c}, \frac{a q}{d e}, \frac{b q}{c}\right)_{\infty}} .
\end{aligned}
$$

A further limit $c \rightarrow \infty$ is easily calculated to give

$$
\begin{aligned}
& { }_{2} \phi_{1}\left(\begin{array}{l}
d, e \\
\frac{a q}{b} ; \frac{a q}{d e}
\end{array}\right){ }_{2} \phi_{1}\left(\begin{array}{l}
\frac{b d}{a}, \frac{b e}{a} ; \frac{a q}{a} \\
\frac{b e}{a}
\end{array}\right) \\
& -\frac{a q}{d e} \frac{(1-e)(1-d)}{\left(1-\frac{b}{a}\right)\left(1-\frac{a q}{b}\right)}{ }_{2} \phi_{1}\left(\begin{array}{c}
d q, e q \\
\left.\frac{a q^{2}}{b} ; \frac{a q}{d e}\right)
\end{array}{ }_{2} \phi_{1}\left(\begin{array}{l}
\frac{b d}{a}, \frac{b e}{a} ; \frac{a q}{a} \\
\frac{b e}{d e}
\end{array}\right)\right. \\
& +\frac{b q}{d e} \frac{\left(1-\frac{b d}{a}\right)\left(1-\frac{b e}{a}\right)}{\left(1-\frac{b}{a}\right)\left(1-\frac{b q}{a}\right)} 2_{2} \phi_{1}\left(\begin{array}{c}
d, e \\
\left.\frac{a q}{b} ; \frac{a q}{d e}\right)
\end{array}{ }_{2} \phi_{1}\left(\begin{array}{c}
\frac{b d q}{a}, \frac{b e q}{a} a \\
\frac{b q^{2}}{a}
\end{array} ; \frac{a q}{d e}\right)\right. \\
& =\frac{(b q)_{\infty}}{\left(\frac{a q}{d e}\right)_{\infty}} \text {. }
\end{aligned}
$$


Even at the ${ }_{2} \phi_{1}$ level, this is not an identity we are familiar with. However, if we now put $d=1$ it reduces to

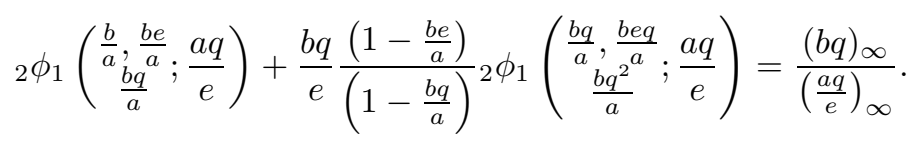

This may now be recognized as the contiguous relation [16]

$$
{ }_{2} \phi_{1}\left(\begin{array}{c}
A, B \\
C
\end{array} ; z\right)+\frac{A z(1-B)}{(1-C)}{ }_{2} \phi_{1}\left(\begin{array}{c}
A q, B q \\
C q
\end{array} ;\right)={ }_{2} \phi_{1}\left(\begin{array}{c}
A q, B \\
C
\end{array} ; z\right)
$$

for the special case $C=A q$ when

$$
{ }_{2} \phi_{1}\left(\begin{array}{c}
A q, B \\
A q
\end{array} ; z\right)={ }_{1} \phi_{0}\left(\begin{array}{l}
B \\
-
\end{array} z\right)=\frac{(B z)_{\infty}}{(z)_{\infty}} .
$$

\section{REFERENCES}

1. R. Askey, Ramanujan and hypergeometric and basic hypergeometric series, Ramanujan International Symposium on Analysis (N. K. Thakare, ed.), MacMillan, India, Delhi, 1989, pp. 1-83. MR 92k:01019

2. R. Askey and J. A. Wilson, Some basic hypergeometric polynomials that generalize Jacobi polynomials, Memoirs Amer. Math. Soc. 319 (1985), 1-55. MR 87a:05023

3. W. N. Bailey, Series of hypergeometric types which are infinite in both directions, Quart. J. Math. (Oxford) 7 (1936), 105-115.

4. __, Generalized Hypergeometric Series, Cambridge University Press, Cambridge, reprinted by Hafner, New York, 1964. MR 32:2625

5. B. C. Berndt, R. L. Lamphere and B. M. Wilson, Chapter 12 of Ramanujan's second notebook: Continued fractions, Rocky Mountain J. Math 15 (1985), 235-310. MR 87k:33003

6. T. S. Chihara, An Introduction to Orthogonal Polynomials, Gordon and Breach, New York, 1978. MR 58:1979

7. G. Gasper and M. Rahman, Basic Hypergeometric Series, Cambridge University Press, Cambridge, 1990. MR 91d:33034

8. W. Gautschi, Computational aspects of three-term recurrence relations, SIAM Rev. 9 (1967), 24-82. MR 35:3927

9. D. P. Gupta and D. R. Masson, Exceptional q-Askey-Wilson polynomials and continued fractions, Proc. Amer. Math. Soc. 112 (1991), 717-727. MR 92b:33047

10. W Watson's basic analogue of Ramanujan's Entry 40 and its generalization, SIAM J. Math. Anal. 25 (1994), 429-440. MR 95b:33047

11. Solutions to the associated q-Askey-Wilson polynomial recurrence relation, Approximation and Computation (R. V. M. Zahar, ed.), Birkhäuser, Boston, 1994, pp. 273-284. MR 97b:33021

12. _ Contiguous relations, continued fractions and orthogonality: an $8 \phi_{7}$ model, J. of Comp. and Appl. Math. 65 (1995), 157-164. MR 97a:33046

13. D. P. Gupta, M. E. H. Ismail and D. R. Masson, Associated continuous Hahn polynomials, Canad. J. Math. 43 (1991), 1263-1280. MR 92m:33016

14. Contiguous relations, basic hypergeometric functions and orthogonal polynomials II, associated big q-Jacobi polynomials, J. Math. Anal. Appl. 171 (1992), 477-497. MR 93k:33011

15. - Contiguous relations, basic hypergeometric functions and orthogonal polynomials III, associated continuous dual q-Hahn polynomials, J. of Comp. and App. Math. 68 (1996), 115149. CMP 97:4

16. M. E. H. Ismail and C. Libis, Contiguous relations, basic hypergeometric functions and orthogonal polynomials, J. Math. Anal. Appl. 141 (1989), 349-372. MR 90k:33010

17. M. E. H. Ismail and D. R. Masson, Generalized orthogonality and continued fractions, J. Approx. Theory 83 (1995), 1-40. MR 97d:42020

18. M. E. H. Ismail and M. Rahman, Associated Askey-Wilson polynomials, Trans. Amer. Math. Soc. 328 (1991), 201-239. MR 92c:33019 
19. W. B. Jones and W. J. Thron, Continued Fractions: Analytic Theory and Applications, Addison-Wesley, Reading, Mass., 1980. MR 82c:30001

20. R. Koekoek and R. F. Swarttouw, The Askey-scheme of hypergeometric orthogonal polynomials and its q-analogue, Reports of the Faculty of Technical Mathematics and Informatics no. 94-05, Delft (1994).

21. D. R. Masson, Some continued fractions of Ramanujan and Meixner-Pollaczek polynomials, Canad. Math. Bull. 32 (1989), 177-181. MR 90i:30007

22. — Wilson polynomials and some continued fractions of Ramanujan, Rocky Mountain J. Math. 21 (1991), 489-499. MR 92h:33015

23. _ Associated Wilson polynomials, Constr. Approx. 7 (1991), 521-534. MR 92h:33014

24. A generalization of Ramanujan's best theorem on continued fractions, Canad. Math. Reports of the Acad. of Sci. 13 (1991), 167-172. MR 92i:11010

25. _ - The last of the hypergeometric continued fractions, Mathematical Analysis and Signal Processing, Contemporary Mathematics (M. E. H. Ismail et. al., eds.), vol. 190, Amer. Math. Soc., Providence, RI, 1995, pp. 287-294. MR 97a:33048

26. M. Rahman and S. K. Suslov, Classical biorthogonal rational functions, Methods of Approximation Theory in Complex Analysis and Mathematical Physics IV (A. A. Gonchar and E. B. Saff, eds.) Lecture Notes in Mathematics 1550, Springer-Verlag, Berlin, 1993, pp. 131146. MR 95m: 42034

27. L. J. Slater, A note on equivalent product theorems, Math. Gazette 38 (1954), 127-128.

28. S. K. Suslov, private communication.

29. G. N. Watson, Ramanujan's continued fraction, Proc. Cambridge Philos. Soc. 31 (1935), 7-17.

30. J. A. Wilson, Hypergeometric Series, Recurrence relations, and Some New Orthogonal Functions, Ph.D. Thesis, University of Wisconsin, Madison, WI, 1978.

31. _ Orthogonal functions from Gram determinants, SIAM J. Math. Anal. 22 (1991), 1147-1155. MR 92c:33018

Department of Mathematics, University of Toronto, Toronto, M5S 3G3, Canada

E-mail address: masson@math.toronto.edu 